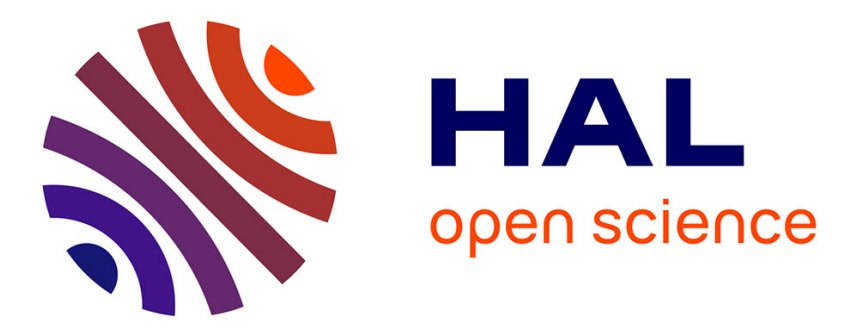

\title{
Boolean dynamics revisited through feedback interconnections
}

\author{
Madalena Chaves, Daniel Figueiredo, Manuel A. Martins
}

\section{To cite this version:}

Madalena Chaves, Daniel Figueiredo, Manuel A. Martins. Boolean dynamics revisited through feedback interconnections. Natural Computing, 2018, 10.1007/s11047-018-9716-8 . hal-01936299

\section{HAL Id: hal-01936299 \\ https://hal.science/hal-01936299}

Submitted on 27 Nov 2018

HAL is a multi-disciplinary open access archive for the deposit and dissemination of scientific research documents, whether they are published or not. The documents may come from teaching and research institutions in France or abroad, or from public or private research centers.
L'archive ouverte pluridisciplinaire HAL, est destinée au dépôt et à la diffusion de documents scientifiques de niveau recherche, publiés ou non, émanant des établissements d'enseignement et de recherche français ou étrangers, des laboratoires publics ou privés. 
This is a preliminary version of the article published as:

M. Chaves, D. Figueiredo, and M.A. Martins, Natural Computing, 2019, https://doi.org/10.1007/s11047-018-9716-8.

\title{
Boolean dynamics revisited through feedback interconnections
}

\author{
Madalena Chaves*, Daniel Figueiredo and Manuel António Martins ${ }^{\dagger}$
}

\begin{abstract}
Boolean models of physical or biological systems describe the global dynamics of the system and their attractors typically represent asymptotic behaviors. In the case of large networks composed of several modules, it may be difficult to identify all the attractors. To explore Boolean dynamics from a novel viewpoint, we will analyse the dynamics emerging from the composition of two known Boolean modules. The state transition graphs and attractors for each of the modules can be combined to construct a new asymptotic graph which will (1) provide a reliable method for attractor computation with partial information; (2) illustrate the differences in dynamical behavior induced by the updating strategy (asynchronous, synchronous, or mixed); and (3) show the inherited organization/structure of the original network's state transition graph.
\end{abstract}

Keywords: Boolean models, feedback interconnections, attractor computation, asynchronous vs. synchronous updates

\section{Introduction}

The effect of network topology in a system's dynamics and stability has long been a problem of interest $[26,12,13]$. In the presence of very large networks of interaction with uncertain knowledge or data involving large maps of activation/inactivation effects, Boolean models with their qualitative On/Off description, provide a practical framework to analyse and model such data $[45,36]$. Therefore, it is very useful to develop new techniques to analyse and render Boolean models more realistic (software [27, 1], asynchronous analysis [5, 17, 40], probabilistic approaches [37, 39], or stochastic approaches [31]).

A Boolean network is defined by a set of logical rules and a strategy for generating the dynamics, such as synchronous or asynchronous updates, or intermediate combinations [43, 21]. The state transititon graph and its dynamics depend not only on the network topology but also on the updating strategy. In fact, different modes of update encompass fundamentally distinct hypotheses regarding the dynamics of the network, and lead to distinct dynamical behavior and attractors $[12,13]$. Synchronous updates are commonly used but they assume simultaneous timescales for all phenomena, and typically generate cyclic attractors which have no biological meaning. Asynchronous updates are arguably more realistic, consider different timescales for the variables in the system, but they have the disadvantage of involving high calculation costs to obtain the state transition graph for large networks (for instance, those containing above 20 vertices).

\footnotetext{
${ }^{*}$ Université Côte d'Azur, Inria, INRA, CNRS, Sorbonne Université, Biocore team, Sophia Antipolis, France, Email: madalena.chaves@inria.fr

${ }^{\dagger}$ CIDMA - Center for R\&D Mathematics and Applications, Department of Mathematics, University of Aveiro, Portugal, Email: daniel.figueiredo@ua.pt,martins@ua.pt
} 
The study of large networks, in particular the computation of its steady states and attractors, remains a hard problem. Several algorithms have been proposed to determine the singletons in a Boolean network, using approaches such as constrained programming [14], SAT formulas for special classes of functions [2], or partitioning the system into smaller networks [23]. Using computational algebra, [42] construct a very cost-efficient algorithm to compute all singletons in a Boolean network. Finding cyclic attractors is an even more complex task and generally needs computation of the successors of all states (typically unfeasible for networks above 20 vertices), or at least the evaluation of certain partial states [24]. The notion of seeds, for representing dynamically closed subspaces, is used in [28, 29] to aproximately compute attractors of a network. A new network reduction method in [44] was shown to be very effective for computation of all types of attractors. Another approach, also based on network partitioning, was proposed by Chaves \& Tournier [8, 9, 40]: this uses a control theory framework to represent a large network as the feedback interconnection between two or more modules and is guaranteed to find not only the singletons but actually all the attractors of the large network, under asynchronous updates.

Some of these methods can be very efficient for analysis of large networks [44, 42], but our aim is to study an algorithm that allows to link together two known models and observe how the new dynamics emerges from each of the two network's attractors. Feedback interconnections are a widely used concept in control theory [38] and essentially mean that two systems with outputs and inputs may be composed into a large system by connecting each system's output to the input of the other system. Interconnection algorithms are especially relevant in physical and biological sciences, when the aim is to understand the behavior of a large system in terms of its different parts, such as the interactions between metabolic and genetic networks [4], or between two fundamental biological modules such as the cell cycle and circadian clock [22]). Simultaneously, new disciplines like synthetic biology aim at the construction of synthetic "biological bricks" that perform a well defined function [34] and can then be assembled together. In this context, the behavior of a system must be analysed in the light of its interactions with other systems (the question of retroactivity [41] may arise), and the study of large networks as the interconnection of one or more smaller modules gains full significance.

The goal of this paper is to revisit the dynamical analysis of Boolean networks in the light of modular feedback interconnections. In particular, the following perspectives will be analysed: (i) develop an algorithm allowing to compute the attractors and asymptotic dynamics for the composition of (already known) networks (see Sections 3, 4, and 5); (ii) understand the dynamical behavior of a network as the feedback interconnection of two modules; gain insight on the structure and organization of the whole state space (see Section 2) and its dependence on the updating strategy. The method proposed in $[8,40]$ is based on the computation of a new object, the asymptotic graph, a directed graph whose vertices are the products of attractors of the two (or more) modules that constitute the Boolean network and the edges are computed from the state transition graphs of these modules. This method is guaranteed to recover all the attractors of the large network (Section 3) but, for very particular cases, new spurious attractors may also be generated. Here, we will first show how to extend this method to avoid spurious attractors (Section 3.2). Second, we will show how to compute an asymptotic graph in the case of synchronous (Section 4) or mixed updating strategies (Section 5). Finally, several examples of interconnections of biological systems will be analyzed to illustrate our methods (Section 7). 


\section{Boolean module feedback interconnections and useful nota- tion}

Our objective is to study the dynamics of Boolean networks and their asymptotic behavior, as emergent properties of the interactions between two smaller separate networks. Throughout this paper, we would like to answer the following questions: How are the pathways constructed, in a Boolean network composed of different modules? How to compute the attractors of the large network, depending on updating strategies?

Here, the focus is on synchronous versus asynchronous strategies, the comparison of their respective dynamical properties, and implications for the computation of the network's pathways and attractors.

Our approach is based on the representation of a Boolean network as the feedback interconnection of two separate Boolean modules. In fact, in physical and biological systems, a larger network is often constructed by putting together smaller, already known modules. To connect the modules, entry nodes and outgoing variables are needed, so we will start by introducing input/output Boolean modules (a notion which is common in control theory [38], for instance).

An input/output Boolean module $\Sigma_{X}=\left(\Omega_{X}, U_{X}, H_{X}, f_{X}, h_{X}\right)$, with $n_{X}$ state variables, $p_{X}$ input variables, and $q_{X}$ output variables is characterized by:

- state space, $\Omega_{X}=\{0,1\}^{n_{X}}$,

- input space $U_{X}=\{0,1\}^{p_{X}}$,

- output space $H_{X}=\{0,1\}^{q_{X}}$,

- set of Boolean rules $f_{X}: \Omega_{X} \times U_{X} \rightarrow \Omega_{X}$,

- output function $h_{X}: \Omega_{X} \rightarrow H_{X}$.

For each $u \in U_{X}$, the synchronous successor of state $x \in \Omega_{X}$ is given by $x^{+}=f_{X}(x ; u)$ and the output associated to this state is $y=h_{X}(x)$.

Given two such modules, $\Sigma_{A}$ and $\Sigma_{B}$, we will use the objects above with subscript $A$ or $B$ and the notation $a \in \Omega_{A}, b \in \Omega_{B}$ for the states of each module. Assuming without loss of generality that $p_{A}=q_{B}$ and $p_{B}=q_{A}$, a larger network can be formed by connecting the input of one system to the output of the other system, i.e., $u_{A}=h_{B}(b)$ and $u_{B}=h_{A}(a)$. The feedback interconnection of the two modules is defined as the system without outputs or inputs $\Sigma_{I}=\left(\Omega_{I}, F_{I}\right)$ with $\Omega_{I}=\{0,1\}^{n_{A}+n_{B}}$, states $x=(a, b)$ and:

$$
F_{I}:=\Omega_{I} \rightarrow \Omega_{I}, \quad F_{I}(x)=F_{I}(a, b)=\left(f_{A}\left(a ; h_{B}(b)\right), f_{B}\left(b ; h_{A}(a)\right)\right),
$$

in other words, the synchronous sucessor of $x=(a, b)$ is $x^{+}=\left(a^{+}, b^{+}\right)$with $a^{+}=f_{A}\left(a ; h_{B}(b)\right)$ and $b^{+}=f_{B}\left(b ; h_{A}(a)\right)$. The dynamical behavior of Boolean model $\Sigma_{I}$ (either synchronous or asynchronous) can be fully described by a state transition graph, $G^{I}$, which can in turn be represented by a $2^{n_{I}} \times 2^{n_{I}}$ matrix. The asymptotic behavior of the network is given by the attractors of $G^{I}$, which are sets of states from which trajectories cannot escape.

In the case of large networks (eg., $n_{I}>20$ ), the exact computation of the graph $G^{I}$ becomes very costly and not feasible in practice. Hence, we propose to characterize the dynamical behavior of $\Sigma_{I}$ from the behavior of the two individual modules $\Sigma_{A}$ and $\Sigma_{B}$, by constructing a new object called the asymptotic graph, $G^{a}$. This new graph is based on a much smaller number of vertices and is less costly to compute. To do this we will first compute their state transition graphs denoted by $G^{A, u}$, one for each input $u \in U_{A}$ and $G^{B, v}$, for each $v \in U_{B}$. These graphs can be constructed either for the asynchronous or synchronous strategies (see Sections 3 and 4, respectively). 


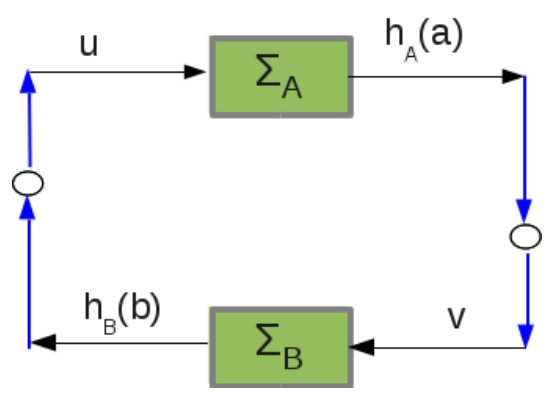

Figure 1: A dynamical system $\Sigma_{I}$ viewed as the feedback interconnection of two modules $\Sigma_{A}$ and $\Sigma_{B}$.

\subsection{Basic definitions and notation}

Some notions used through the text are now recalled (see, for instance, [15]). A directed graph, $G=(\mathcal{V}, \mathcal{E})$ is defined by a set of vertices, $\mathcal{V}$ and a set of directed edges, $\mathcal{E}$, where a directed edge is a pair of ordered vertices, $V_{1} \rightarrow V_{2}$.

A directed graph is strongly connected if any pair of vertices in $V$ is connected by a path (where a path is a sequence of distinct edges $V_{1} \rightarrow V_{2} \rightarrow \cdots V_{i}$ ). Any directed graph $G$ can be organized into strongly connected components (SCCs), where an SCC is a maximal strongly connected subgraph of $G$. An SCC can contain either a single vertex or a subset of vertices (it can also contain the full graph). An SCC $C$ can have incoming or outgoing pathways, that is a pathway from (or towards) a vertex $V \notin C$. An SCC without any outgoing pathways is called an attractor. In other words, when a trajectory reaches an attractor it remains inside for all subsequent updating times and, therefore, distinct attractors represent distinct asymptotic behaviors of the system.

The set of attractors of a given graph $G$ is denoted $\mathcal{A}(G)$. The reachable set of a vertex $a$ in $G, \mathcal{R}(a ; G)$, is the set of all vertices $\tilde{a}$ such that there exists a path connecting $a$ to $\tilde{a}$ in $G, a \stackrel{G}{\sim} \tilde{a}$. The reachable set of a subset $S \subset \mathcal{V}$ in $G$ is the union $\mathcal{R}(S ; G)=\cup_{a \in S} \mathcal{R}(a ; G)$. Conversely, the basin of attraction of $A \in \mathcal{A}(G)$ in $G, \mathcal{B}(A ; G)$, is the set of all vertices a such that there exists a path connecting it to $A$ in $G, a \stackrel{G}{\sim} A$.

For a system $\Sigma_{X}$ with input $\nu$, the state transition graph is a directed graph, denoted by $G^{X, \nu}=\left(\Omega_{X}, \mathcal{E}_{X}\right)$ where the set of edges is computed from the Boolean rules $f_{X}$ and the updating strategy. To facilitate notation, $\nu$ takes values in lexicographic order, i.e., $\nu \in$ $\left\{1,2, \ldots, 2^{p_{X}}\right\}$ where " $1=0 \ldots 00 ", " 2=0 \ldots 01 "$, and " $2^{p_{X}}=11 \cdots 1 "$.

For each module $\Sigma_{X}$, the state transition graphs $G^{X, \nu}$ will have $L^{X, \nu} \geq 1$ attractors denoted $X_{\nu}^{i}$. To avoid introducing heavy notation, we will use $a \in A_{u}^{i}$ (or $b \in B_{v}^{j}$ ) to name the elements of $G^{A, u}$ (or $G^{B, v}$ ) contained in each attractor.

Table 1 summarizes the notation used for the state transition graphs and attractors for different modules and updating strategies.

\section{$2.2 \quad$ A working example}

To illustrate our methodology and the differences generated by the three updating strategies, consider the interconnection between two modules, one negative loop and one double-negative loop:

$$
\begin{array}{ll}
\Sigma_{A}: & a_{1}^{+}=\neg a_{2}, \quad a_{2}^{+}=\neg u \wedge \neg a_{1}, \quad h_{A}(a)=\left(a_{1}, a_{2}\right)^{\prime}, \\
\Sigma_{B}: & b_{1}^{+}=v_{1} \wedge \neg b_{2}, \quad b_{2}^{+}=\neg v_{2} \wedge b_{1}, \quad h_{B}(b)=b_{2},
\end{array}
$$


Table 1: Summary of notation used throughout the paper to name state transition graphs. Three updating strategies will be compared for the analysis of the interconnection of two Boolean modules. These yield different transition graphs for each module and for the full network and asymptotic graphs. In the mixed strategy, the modules are synchronously updated. In general, a subscript $*$ will be used for asynchronous updates, $m x$ will be used for a mixed strategy, and no subscript for synchronous updates. To avoid an overcharged notation, the semi-attractors are similarly labeled for both asynchronous and synchronous updates, but this is usually clear from the context.

\begin{tabular}{|c|c|c|c|c|c|c|}
\hline \multirow[t]{2}{*}{ Updates } & \multicolumn{2}{|c|}{ Modules } & \multicolumn{2}{|c|}{ Full network } & \multicolumn{2}{|c|}{ Asymptotic graph } \\
\hline & graphs & semi-attractors & graph & attractors & graph & tractors \\
\hline Synchronous & $\overline{\bar{G}^{A, u}, G^{B, v}}$ & $A_{u \alpha}^{i}, B_{v \beta}^{j}$ & $G^{I}$ & $Q^{k}$ & $G^{a}$ & $Q^{a, k}$ \\
\hline Mixed & $G^{A, u}, G^{B, v}$ & $A_{u \alpha}^{i}, B_{v \beta}^{j}$ & $G_{m x}^{I}$ & $Q_{m x}^{k}$ & $G_{m x}^{a}$ & $Q_{m x}^{a, k}$ \\
\hline Asynchronous & $G_{*}^{A, u}, G_{*}^{B, v}$ & $A_{u \alpha}^{i}, B_{v \beta}^{j}$ & $G_{*}^{I}$ & $Q_{*}^{k}$ & $G_{*}^{a}$ & $Q_{*}^{a, k}$ \\
\hline
\end{tabular}

where $N_{A}=N_{B}=2, p_{A}=q_{B}=2, p_{B}=q_{A}=1$. The interconnected system $\Sigma_{I}$ is obtained by setting

$$
u=b_{2}, \quad v_{1}=a_{1}, \quad v_{2}=a_{2} .
$$

The state transition graphs under an asynchronous or a synchronous strategy are shown in Fig. 2. The graphs $G^{a}$ or $G^{I}$ will be constructed throughout the following sections.
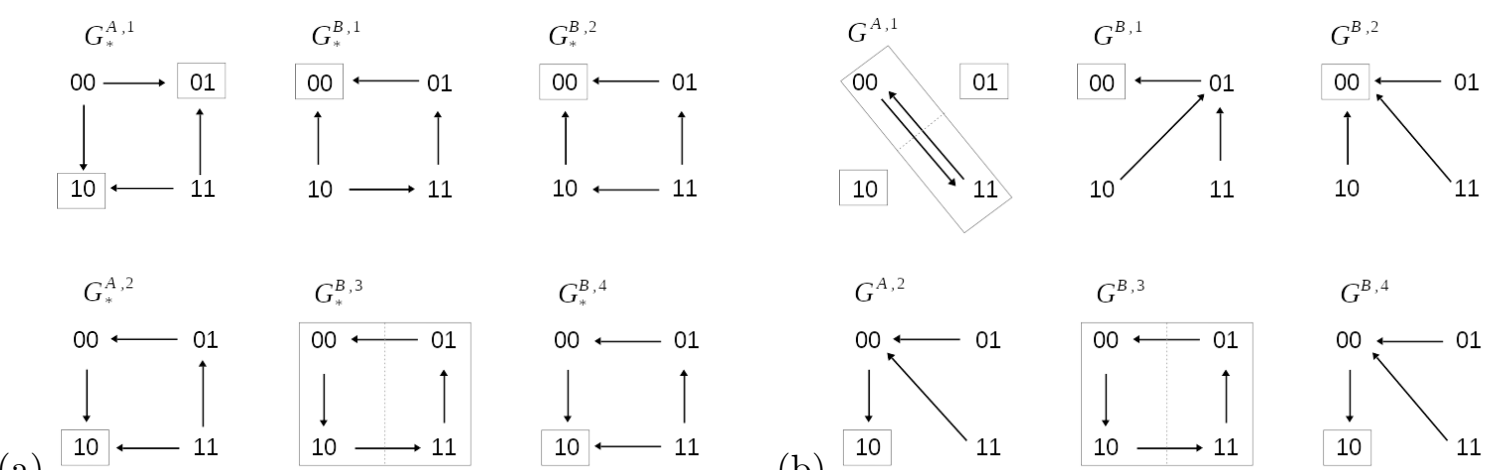

(a)

Figure 2: The state transition graphs for modules $\Sigma_{A}$ and $\Sigma_{B}$ of (2), using (a) asynchronous or (b) synchronous updates.

\section{The asynchronous asymptotic graph method for attractor computation}

Once the attractors of all the transition graphs are known, a new object will be constructed, called the asymptotic graph, $G_{*}^{a}$, a graph where the vertices are basically all products of attractors of the form $A_{u}^{i} \times B_{v}^{j}$ and the edges are computed based on the transition graphs $G_{*}^{A, u}$ and $G_{*}^{B, v}$. The $G_{*}^{a}=\left(\mathcal{V}_{*}, \mathcal{E}_{*}\right)$ is constructed differently depending on the updating strategy and is next detailed. Note that this method can be generalized to the interconnection between three or more modules by appropriately defining input and output functions (see [40]). For simplicity, we will discuss only the interconnection between two modules. 
The main results in this paper establish that there is a correspondence between $\mathcal{A}\left(G_{*}^{a}\right)$ and $\mathcal{A}\left(G_{*}^{I}\right)$ which depends on the updating strategy.

The asymptotic graph for asynchronous updates was first proposed in [8] and then further studied in $[40,6]$. This Section briefly reviews the main results.

\subsection{Step-by-step construction}

The asynchronous strategy consists in changing exactly one coordinate at each time step. More precisely, fix $u$, for each state compute $x^{+}=f_{X}(x ; u)$ and define the set of indices whose coordinates change:

$$
\mathcal{I}(x ; u)=\left\{i: x_{i}^{+}-x_{i} \neq 0\right\}
$$

Then, the possible asynchronous successors of $x$ in $G_{*}^{X, u}$ are:

$$
y(x ; u) \in\left\{\hat{x}: \hat{x}_{i}=1-x_{i}, \text { for some } i \in \mathcal{I}(x ; u), \hat{x}_{j}=x_{j}, j \neq i\right\} .
$$

Step 1: Construct the set of vertices $\mathcal{V}_{*} \quad$ The first step of the asympotic graph method is to divide each attractor of the modules into subsets of states that have the same output. These are called semi-attractors of the modules and are defined as follows. Given $A_{u}^{\bar{\imath}} \in \mathcal{A}\left(G_{*}^{A, u}\right)$ and $B_{v}^{\bar{\jmath}} \in \mathcal{B}\left(G_{*}^{B, v}\right)$, define:

$$
\begin{aligned}
& A_{u \alpha}^{i}=\left\{a \in A_{u}^{\bar{\tau}}: h_{A}(a)=\operatorname{bool}(\alpha)\right\} \\
& B_{v \beta}^{j}=\left\{b \in B_{v}^{\bar{j}}: h_{B}(b)=\operatorname{bool}(\beta)\right\}
\end{aligned}
$$

where the superscripts $i, j$ range over the total number of semi-attractors for the module (over all inputs), $i \in\left\{1, \ldots, L_{*}^{A}\right\}, j \in\left\{1, \ldots, L_{*}^{B}\right\}$. (Note that $i$ and $\bar{\imath}$ are not related but we have $u=u(i)$ and $\alpha=\alpha(i)$; we drop these dependences to simplify notation. A similar observation holds for $j$.) The subscripts range over $v, \alpha \in\left\{1, \ldots, 2^{p_{B}}\right\}$ and $u, \beta \in\left\{1, \ldots, 2^{p_{A}}\right\}$, and bool $(\nu)$ denotes the Boolean representation of integer $\nu$. Observe that some of the semi-attractors may be empty and are neglected. As an example, the counting of semi-attractors for system (2) is given in (6) below.

The vertices of $G_{*}^{a}$ are the cross-products of non-empty semi-attractors:

$$
\mathcal{V}_{*}=\left\{A_{u \alpha}^{i} \times B_{v \beta}^{j}: i=1, \ldots, L_{*}^{A}, j=1, \ldots, L_{*}^{B}\right\},
$$

where $L_{*}^{A}$ and $L_{*}^{B}$ are the total number of semi-attractors for modules $A$ and $B$ (respectively), under asynchronous updates (and the dependences $u=u(i), \alpha=\alpha(i), v=v(j), \beta=\beta(j)$ are omitted for simplicity).

Step 2: Construct the set of edges $\mathcal{E}_{*}$ To satisfy an asynchronous updating strategy, each edge corresponds to the update of a single module, i.e., only the $a$ (or $b$ ) variables are allowed to change at each edge. Accordingly, only edges of the following form are considered

$$
A_{u \alpha}^{i} \times B_{v \beta}^{j} \rightarrow A_{u \alpha}^{i} \times B_{\alpha \tilde{\beta}}^{\tilde{\jmath}} \quad \text { or } \quad A_{u \alpha}^{i} \times B_{v \beta}^{j} \rightarrow A_{\beta \tilde{\alpha}}^{\tilde{\imath}} \times B_{v \beta}^{j}
$$

such that one of the modules is fixed at a semi-attractor, while the dynamics of the other module evolves: in the first case, the semi-attractor of $G_{*}^{A, u}$ is fixed while the dynamics of $\Sigma_{B}$ follows the path(s) of $B_{v \beta}^{j}$ along the graph with input $v=\alpha, G_{*}^{B, \alpha}$. Therefore, there is an edge between the two vertices if there is a path in the graph $G_{*}^{B, \alpha}$ that leads from some state in 
$B_{v \beta}^{j}$ to some state in $B_{\alpha \tilde{\beta}}^{\tilde{\jmath}}$. Similarly for an edge $A_{u \alpha}^{i} \times B_{v \beta}^{j} \rightarrow A_{\beta \tilde{\alpha}}^{\tilde{q}} \times B_{v \beta}^{j}$. The set of edges can be written:

$$
\mathcal{E}_{*}=\left\{(V, W) \in \mathcal{V}_{*}: V=A_{u \alpha}^{i} \times B_{v \beta}^{j} \text { and }\left(W=A_{u \alpha}^{i} \times B_{\alpha \tilde{\beta}}^{\tilde{\jmath}} \text { or } W=A_{\beta \tilde{\alpha}}^{\tilde{u}} \times B_{v \beta}^{j}\right)\right\},
$$

where $A_{\beta \tilde{\alpha}}^{\tilde{\imath}}$ is in the reachable set of $A_{u \alpha}^{i}$ in the graph $G_{*}^{A, \beta}$ and $B_{\alpha \tilde{\beta}}^{\tilde{j}}$ is in the reachable set of $B_{v \beta}^{j}$ in the graph $G_{*}^{B, \alpha}$.

Step 3: Attractor computation In the asynchronous case, $\mathcal{A}\left(G_{*}^{a}\right)$ contains a representative of each of the attractors of $G_{*}^{I}$ as was shown in $[8,40]$ :

Theorem 1 [8] In the case of asynchronous networks, if $Q_{*}$ is an attractor of $G_{*}^{I}$, then there exists at least one corresponding attractor in $G_{*}^{a}, Q_{*}^{a}=Q_{*}^{a}\left(Q_{*}\right)$. Moreover, if $Q_{*}^{1} \neq Q_{*}^{2}$ are two distinct attractors of $G_{*}^{I}$, then $Q_{*}^{a, 1}\left(Q_{*}^{1}\right) \neq Q_{*}^{a, 2}\left(Q_{*}^{2}\right)$.

Therefore, all the attractors of the asynchronous $G_{*}^{I}$ can be obtained from the attractors of $G_{*}^{a}$. However, it may happen that some of the attractors of $G_{*}^{a}$ are not true attractors of $G_{*}^{I}$, also called "spurious" attractors. The formation of such attractors is illustrated with the mixed updating strategy (Section 5).
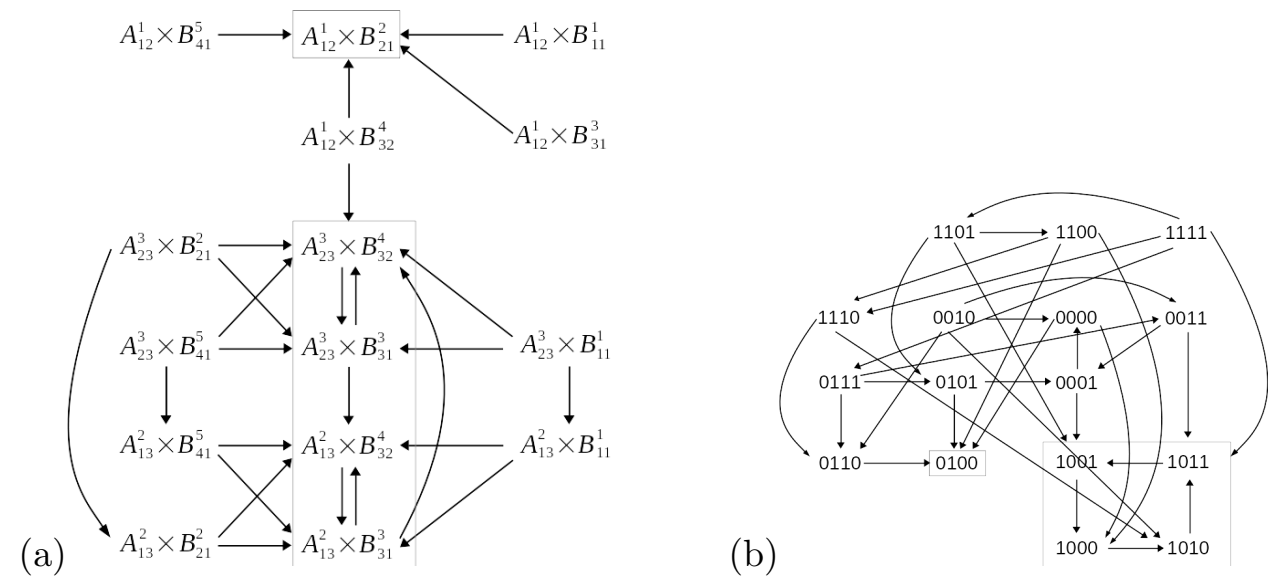

Figure 3: (a) The asynchronous asymptotic graph and (b) the full network state transition graph for the interconnection of modules $\Sigma_{A}$ and $\Sigma_{B}$ in (2).

Example For the network (2), the corresponding asynchronous graphs $G_{*}^{A, u}$ and $G_{*}^{B, u}$ are shown in Fig. 2(a) and the sets of semi-attractors are:

$$
\begin{array}{ll}
\mathcal{A}\left(G_{*}^{A, 1}\right): & A_{12}^{1}=\{01\}, A_{13}^{2}=\{10\} \\
\mathcal{A}\left(G_{*}^{A, 2}\right): & A_{23}^{3}=\{10\} \\
\mathcal{A}\left(G_{*}^{B, 1}\right): & B_{11}^{1}=\{00\} \\
\mathcal{A}\left(G_{*}^{B, 2}\right): & B_{21}^{2}=\{00\} \\
\mathcal{A}\left(G_{*}^{B, 3}\right): & B_{31}^{3}=\{00,10\}, B_{32}^{4}=\{01,11\} \\
\mathcal{A}\left(G_{*}^{B, 4}\right): & B_{41}^{5}=\{10\} .
\end{array}
$$

All semi-attractors are also true attractors, except for $B_{31}^{3}$ and $B_{32}^{4}$ each containing the states with same output of the attractor $\{00,01,10,11\}$ of $G_{*}^{B, 3}$. There are thus $3 \times 5=15$ vertices in $\mathcal{V}_{*}$, corresponding to all possible products of semi-attractors $A_{u \alpha}^{i}$ and $B_{v \beta}^{j}$. 
To compute the edges according to (5) consider the vertex $A_{23}^{3} \times B_{41}^{5}$, for instance, which has three outgoing edges. First, fix the part $A_{23}^{3}$, which is "forcing" system $\Sigma_{B}$ with input 3 ; hence we need to compute the successor of $B_{41}^{5}=\{10\}$ in the state transition graph $G_{*}^{B, 3}$. This graph contains a cyclic attractor, divided into two semi-attractors (see Fig. 2(a)) so trajectories of system $\Sigma_{B}$ cycle between these two, $B_{31}^{3}$ and $B_{33}^{4}$. This gives the two edges $A_{23}^{3} \times B_{41}^{5} \rightarrow A_{23}^{3} \times B_{31}^{3}$ and $A_{23}^{3} \times B_{41}^{5} \rightarrow A_{23}^{3} \times B_{32}^{4}$.

Second, fix the part $B_{41}^{5}$ which is forcing system $\Sigma_{A}$ with input 1 ; the only successor of state $A_{23}^{3}=\{10\}$ in $G_{*}^{A, 1}$ is $A_{13}^{2}=\{10\}$.vThis gives an edge $A_{23}^{3} \times B_{41}^{5} \rightarrow A_{13}^{2} \times B_{41}^{5}$.

In this way, obtain the asymptotic graph shown in Fig. 3(b), which has two attractors:

$$
\begin{aligned}
& Q_{*}^{a, 1}=\left\{A_{12}^{1} \times B_{21}^{2}\right\}=\{0100\} \\
& Q_{*}^{a, 2}=\left\{A_{13}^{2} \times B_{31}^{3}, A_{13}^{2} \times B_{32}^{4}, A_{23}^{3} \times B_{31}^{3}, A_{23}^{3} \times B_{32}^{4}\right\}=\{1000,1010,1001,1011\} .
\end{aligned}
$$

Comparison with $G_{*}^{I}$ (Fig. 3(a)) shows that the asymptotic graph exactly recovers the two attractors of the full interconnected system, as expected.

\subsection{Extending the asynchronous asymptotic graph, $G_{*}^{e x t}$}

In the case of the asynchronous asymptotic graph, to improve its construction and avoid the generation of spurious attractors, the idea is to extend the vertex set $\mathcal{V}_{*}$ to include further components. This new set will be called $\overline{\mathcal{V}}_{*}$ and the new asymptotic graph is denoted $G_{*}^{\text {ext }}$.

An analysis of asynchronous asymptotic graphs with failures shows that spurious attractors may appear due to output changes along the pathways followed by the states of an attractor $A_{u}^{i}$ in a state transition graph $G_{*}^{A, \tilde{u}}$ with $\tilde{u} \neq u$ (see the Example in Section 5). These pathways and the corresponding sequence of outputs "forgotten" in the construction of $G_{*}^{a}$ (which keeps only the final state of the pathway, by definition).

To identify the appropriate new components, the idea is to analyze the pathways of each state of each attractor $X_{\nu}^{i}$ along the other partial graphs $G_{*}^{X, \tilde{\nu}}$, with $\tilde{\nu} \neq \nu$, until a change in the output is detected, as follows (for clarity, the case of module $X=A$ will be described):

(i) Compute the strongly connected components (SCCs) of each state transition graph $G_{*}^{A, u}$ and its terminal SCCs (or attractors). Collect its attractors in $\mathcal{A}_{u}=\left\{A_{u}^{i}: i=\right.$ $\left.1, \ldots, L^{A, u}\right\}$.

(ii) Pick an attractor $A_{u}^{i}$, suppose it contains the states $\left\{r_{1}, \ldots, r_{k}\right\}$, and look at all the possible forward pathways for each $r_{\ell}$ in the other graphs $G_{*}^{A, \tilde{u}}$ with $\tilde{u} \neq u$, for instance,

$$
P_{\ell_{1}}: r_{\ell} \rightarrow s_{1} \rightarrow s_{2} \rightarrow \cdots, \quad \text { in } G_{*}^{A, \tilde{u}}
$$

Along each path, each state has its own output $h_{A}\left(s_{i}\right)$.

(iii) For each pathway $P_{\ell_{k}}$ of each state $r_{\ell}$, pick the first state $s_{\hat{\imath}}$ that has a different output from $r_{\ell}$, that is $h_{A}\left(s_{\hat{\imath}}\right)=\tilde{\alpha} \neq h_{A}\left(r_{\ell}\right)=\alpha$.

(iv) This state $s_{\hat{\imath}}$ belongs to some strongly connected component $S_{u}$ of the graph $G_{*}^{A, u}$. Add this SCC to the set of attractors: $\mathcal{A}_{u}=\mathcal{A}_{u} \cup\left\{S_{u}\right\}$.

Repeat steps (i)-(iv) for all $u \in\left\{1, \ldots, 2^{p_{A}}\right\}$ and then for module $B$, for all $v \in\left\{1, \ldots, 2^{p_{B}}\right\}$, to produce the extended sets $\mathcal{A}_{u}$ and $\mathcal{B}_{v}$. The next step is to divide each component of these sets into semi-SCCs with the same output: $\bar{A}_{u \alpha}^{i}=\left\{a \in \bar{A}_{u}^{\bar{\imath}}: h_{A}(a)=\operatorname{bool}(\alpha)\right\}$ and $\bar{B}_{v \beta}^{j}=\left\{b \in \bar{B}_{v}^{\bar{j}}: h_{B}(b)=\operatorname{bool}(\beta)\right\}$. Collecting all the semi-SCCs into:

$$
\mathcal{S}_{A}=\left\{\bar{A}_{u \alpha}^{i}: i=1, \ldots, \bar{L}^{A}\right\}, \quad \mathcal{S}_{B}=\left\{\bar{B}_{v \beta}^{j}: j=1, \ldots, \bar{L}^{B}\right\},
$$


the set of vertices for the extended asynchronous asymptotic graph is thus:

$$
\overline{\mathcal{V}}_{*}=\left\{\bar{A}_{u \alpha}^{i} \times \bar{B}_{v \beta}^{j}: i=1, \ldots, \bar{L}^{A}, j=1, \ldots, \bar{L}^{B}\right\} .
$$

The new set of edges $\overline{\mathcal{E}}_{*}$ is computed as before (5).

To test this method, we applied it to 750 randomly generated pairs of modules. The modules have $N_{A}=N_{B} \in\{2,3,4,5,6\}$ and inputs $p_{A}=p_{B} \in\{1,2,3\}$. Each module was obtained by randomly generating the Boolean truth tables for $G_{*}^{X, \nu}, \nu \in\left\{1, \ldots, 2^{p_{X}}\right\}$. The results obtained with this extended method (Fig. 4) indicate that the spurious attractor problem is solved for about $99.8 \%$ of the cases. However, the new $G_{*}^{e x t}$ is still not exact and it is much larger to calculate, since $\left|G_{*}^{e x t}\right|>\left|G_{*}^{I}\right|$, except for modules with dimension above $N_{X}=6$.

These results also suggest that the addition of other SCCs to the sets $\mathcal{S}_{A}$ and $\mathcal{S}_{B}$ will further improve the asymptotic graph. For instance, by successively adding the SCCs corresponding to a second or third change of output along the pathways $P_{\ell_{k}}$.

Indeed, this joins the result shown in [40] for another graph construction, called the crossgraph, $G^{c r}$ : this graph is contructed from all pairs of all SCCs of $G_{*}^{X, \nu}$. A theorem shows that there is a bijection between the attractors of $G^{c r}$ and $G_{*}^{I}$ (i.e. $G^{c r}$ recovers exactly all attractors of $\left.G_{*}^{I}\right)$. This is not surprising, since $G^{c r}$ virtually contains all states of $G_{*}^{I}$.

The difficulty is that $G^{c r}$ (and possibly other extensions of $G_{*}^{e x t}$ ) is as costly to compute as the graph $G_{*}^{I}$ itself, so it is not an efficient method for large networks. Nevertheless, the successive constructions $G_{*}^{a}, G_{*}^{e x t}, \ldots, G^{c r}$ provide a clear illustration of the organization of asynchronous dynamical behavior.
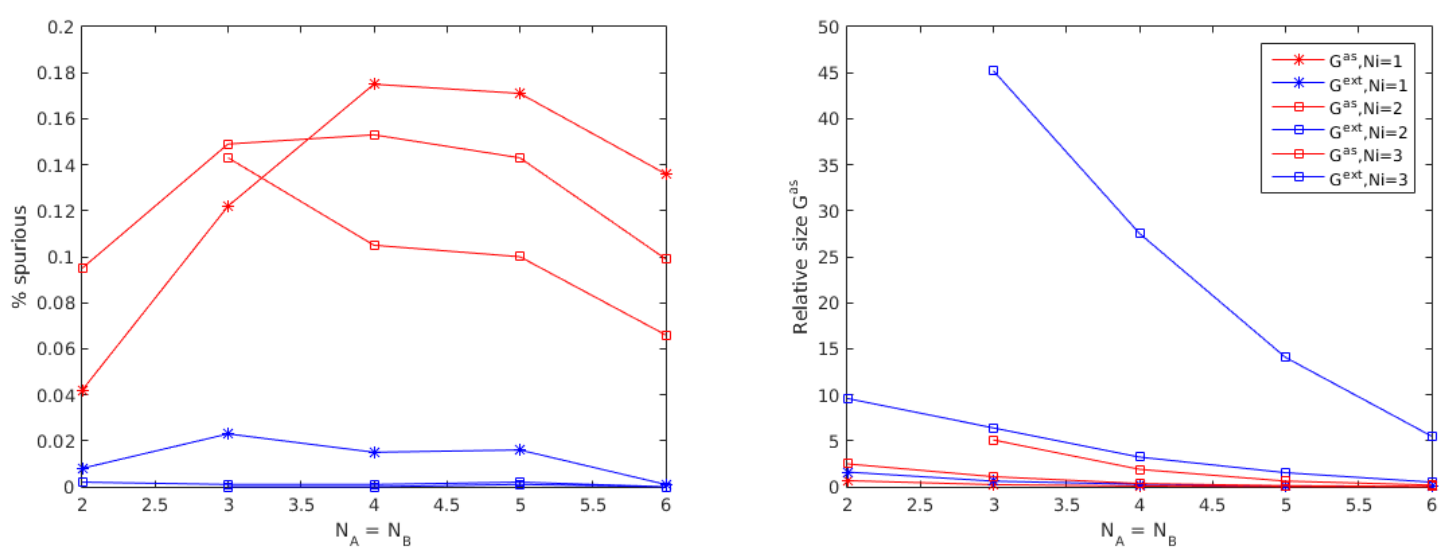

Figure 4: Performance of the extended asymptotic graph method for asynchronous networks (darker curves, $G^{e x t}$ ) and comparison with original method (lighter curves, $G_{*}^{a}$ ), for randomly generated modules, and interconnected networks with $p_{A}=1,2,3$. Left: the percentage of failure (i.e., the generation of spurious). Right: the sizes of both asymptotic graphs relative to the size of the full network $G_{*}^{I}$.

\section{The synchronous asymptotic graph, $G^{a}$}

To construct the asymptotic graph in the synchronous case, one could follow a similar reasoning and define the edges of the asymptotic graph by asking that both the $a$ and $b$ states change simultaneously:

$$
\left(A_{u \alpha}^{i}, B_{v \beta}^{j}\right) \rightarrow\left(A_{\beta \tilde{\alpha}}^{\tilde{\imath}}, B_{\alpha \tilde{\beta}}^{\tilde{\jmath}}\right)
$$


However, a few examples showed that this strategy may not only generate spurious attractors (as in the asynchronous case), but also miss finding some of the attractors of the synchronous $G^{I}$. This is therefore not a favorable strategy for constructing a synchronous asymptotic graph.

To improve the construction and guarantee some result, the asymptotic graph can be constructed according to an iterative procedure, as follows.

Step 1: Construct the set of vertices $\mathcal{W}$ The initial set of vertices is $\mathcal{V}$, constructed as in the asynchronous case (4). Then, given a set of nodes $\mathcal{W}_{k}$, compute their direct successors according to (1) to form $\mathcal{W}_{k+1}$ :

$$
\begin{aligned}
& \mathcal{W}_{0}:=\left\{A_{u \alpha}^{i} \times B_{v \beta}^{j}: i=1, \ldots, L^{A}, j=1, \ldots, L^{B}\right\}, \\
& \mathcal{W}_{k+1}:=\left\{W: W=\left(a^{+}, b^{+}\right), \text {for }(a, b) \in V, \text { all } V \in \mathcal{W}_{k}\right\} \backslash\left(\cup_{\ell=0}^{k} \mathcal{W}_{\ell}\right)
\end{aligned}
$$

where $L^{A}, L^{B}$ are the total number of semi-attractors of modules $A$ and $B$ (respectively) under synchronous updates, and $a^{+}=f_{A}\left(a ; h_{B}(b)\right)$ and $b^{+}=f_{B}\left(b ; h_{A}(a)\right)$. (Since the state space is finite there will eventually be some $\hat{k}$ such that $\mathcal{W}_{\hat{k}}=\emptyset$.) The set of nodes for the synchronous asymptotic graph $G^{a}$ is then:

$$
\mathcal{W}=\cup_{k=0}^{\hat{k}-1} \mathcal{W}_{k}
$$

Step 2: Construct the set of edges $\mathcal{E}$ The set of edges is based on synchronous updates:

$$
\mathcal{E}=\left\{(V, W) \in \mathcal{W}: V=(a, b) \text { and } W=\left(a^{+}, b^{+}\right), V, W \in \mathcal{W}\right\}
$$

As a last remark, in theory, the graph $G^{a}$ may be as long to compute as the full graph $G^{I}$. This is however not the case in practice (see examples below).

Step 3: Attractor computation In the synchronous case, since $G^{a}$ is a subgraph of $G^{I}$, all attractors of $G^{a}$ are guaranteed to be true attractors of $G^{I}$. It may, however, happen that not all attractors of the full network are recovered, in contrast to the asynchronous case.

Theorem 2 In the case of synchronous networks, if $Q^{a}$ is an attractor of $G^{a}$, then $Q^{a}$ contains an attractor of $G^{I}$.

Proof: Let $Q^{a}$ be an attractor of $G^{a}$ and let $\mathcal{B}\left(Q^{a}\right)$ represent its basin of attraction. Then, by definition of the synchronous asymptotic graph, there is at least one element of $\Omega_{I}$ of the form $V=\left(A_{u \alpha}^{i}, B_{v \beta}^{j}\right) \in \mathcal{B}\left(Q^{a}\right)$ (since the set of vertices of $G^{a}$ contains $\left.\mathcal{W}_{0},(7)\right)$. Again, by definition of the synchronous asymptotic graph, all reachable states from $V$ in $G^{I}, \mathcal{R}\left(V ; G^{I}\right)$, are also contained in $\mathcal{W}$ and, namely, $Q^{a}$ contains the attractor corresponding to the path in $\mathcal{R}\left(V ; G^{I}\right)$. In other words, $Q^{a}$ contains an attractor of $G^{I}$.

Example For the network (2), the synchronous transition graphs $G^{A, u}$ and $G^{B, u}$ are shown in Fig. 2(b) and the sets of semi-attractors are:

$$
\begin{array}{ll}
\mathcal{A}\left(G^{A, 1}\right): & A_{11}^{1}=\{00\}, A_{14}^{2}=\{11\}, A_{12}^{3}=\{01\}, A_{13}^{4}=\{10\} \\
\mathcal{A}\left(G^{A, 2}\right): & A_{23}^{5}=\{10\} \\
\mathcal{A}\left(G^{B, 1}\right): & B_{11}^{1}=\{00\} \\
\mathcal{A}\left(G^{B, 2}\right): & B_{21}^{2}=\{00\} \\
\mathcal{A}\left(G^{B, 3}\right): & B_{31}^{3}=\{00,10\}, B_{32}^{4}=\{01,11\} \\
\mathcal{A}\left(G^{B, 4}\right): & B_{41}^{5}=\{10\} .
\end{array}
$$




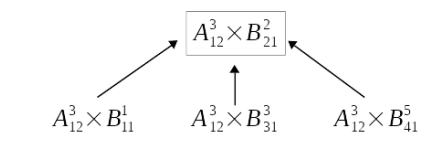

(a)

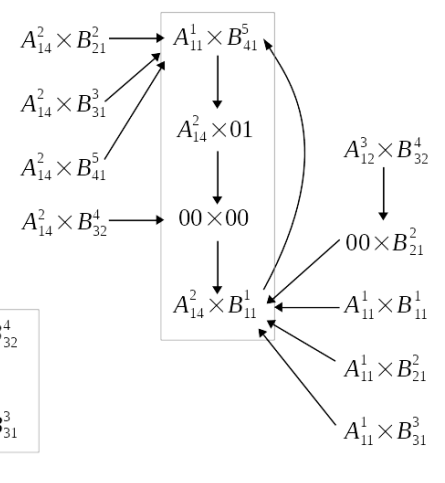

(b)

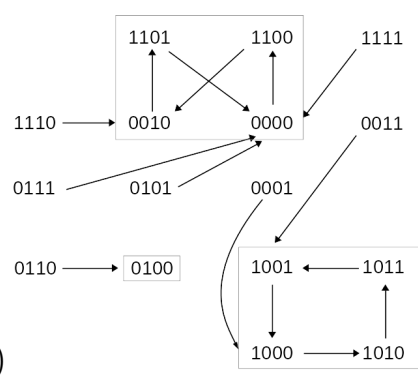

Figure 5: (a) The synchronous asymptotic graph and (b) the full network state transition graph for the interconnection of modules $\Sigma_{A}$ and $\Sigma_{B}$ in (2).

All semi-attractors are also true attractors, except for two pairs $A_{11}^{1} \cup A_{14}^{2}=\{00,11\}$ and $B_{31}^{3} \cup B_{32}^{4}=\{00,01,10,11\}$ organized into same-output semi-attractors. There are thus $5 \times 5=$ 25 vertices in $\mathcal{V}$. According to the iterative procedure $(7)$, the following four vertices need to be added: $00 \times 00,00 \times B_{21}^{2}, A_{14}^{2} \times 01, A_{23}^{5} \times 01$. Fig. $5(\mathrm{a})$, represents depicts the synchronous asymptotic graph which has three attractors:

$$
\begin{aligned}
& Q^{a, 1}=A_{12}^{3} \times B_{21}^{2}=\{0100\} \\
& Q^{a, 2}=\left\{A_{11}^{1} \times B_{41}^{5}, A_{14}^{2} \times 01, A_{14}^{2} \times B_{11}^{1}, 00 \times 00\right\}=\{0000,0010,1100,1101\} \\
& Q^{a, 3}=\left\{A_{13}^{4} \times B_{32}^{4}, A_{23}^{5} \times B_{31}^{3}\right\}=\{1000,1010,1001,1011\} .
\end{aligned}
$$

Comparison with $G^{I}$ (Fig. 5(b)) shows that the synchronous asymptotic graph exactly recovers the attractors of the full interconnected system.

\section{The asymptotic graph, for mixed strategy networks, $G_{m x}^{a}$}

The results in Sections 3 and 4 suggest a natural application of the asymptotic graph method for mixed-strategy networks. Assume that a network $\Sigma_{M}$ with graph $G_{m x}^{I}$ is synchronous in blocks, for instance composed of two groups of variables, $x=(a, b)$, and within each group $a_{1}, \ldots, a_{k}$ or $b_{1}, \ldots, b_{\ell}$ the variables are synchronously updated. The choice to update either group $a$ or group $b$ is according to an asynchronous strategy.

To apply the asymptotic graph method, it is intuitive to partition the network into two modules, each containing one of the synchronous blocks of variables. For each module compute the synchronous graphs $G^{X, u}$ and their corresponding attractors $\mathcal{A}\left(G^{X, u}\right)$. Using these attractors, construct the asymptotic graph as in the asynchronous case, $G_{m x}^{a}=\left(\mathcal{V}_{m x}, \mathcal{E}_{m x}\right)$.

Then, the attractors of $G_{m x}^{a}$ will contain (a representative of) all attractors of $G_{m x}^{I}$ : $\mathcal{A}\left(G_{m x}^{a}\right) \supset \mathcal{A}\left(G_{m x}^{I}\right)$. Intuitively, this is because the attractors of $G^{X, u}$ represent each basin of attraction of each of the synchronous blocks.

To facilitate the analysis, we now introduce a projection function, $\pi: \mathcal{A}\left(G^{X, u}\right) \rightarrow \Omega_{X}$ which returns all the states contained in a given attractor of $G^{X, u}$ and a grouping function, $\psi: \mathcal{A}\left(G_{m x}^{I}\right) \rightarrow \mathcal{V}_{m x}$, which returns all the vertices of $G_{m x}^{a}$ "contained" in an attractor of $G_{m x}^{I}$ :

$$
\psi(Q)=\left\{\left(A_{u \alpha}, B_{v, \beta}\right) \in \mathcal{V}_{m x}:(a, b) \in Q \text { where } a \in \pi\left(A_{u \alpha}\right), b \in \pi\left(B_{v, \beta}\right)\right\} .
$$

The equivalent of Theorem 1 for a mixed block-synchronous strategy can now be stated. The proof follows the same argument as in [8]. 
Theorem 3 If $Q$ is an attractor of $G_{m x}^{I}$, then $\psi(Q)$ contains an attractor of $G_{m x}^{a}$.

Proof: Let $(a, b) \in Q$ with $a$ a vertex of $\Sigma_{A}$ and $b$ a vertex of $\Sigma_{B}$. Let $\alpha=h_{A}(a), \beta=h_{B}(b)$ and consider the attractors $A_{\beta \alpha_{0}}, B_{\alpha \beta_{0}}$ such that $a \in \mathcal{B}\left(A_{\beta \alpha_{0}} ; G^{A, \beta}\right), b \in \mathcal{B}\left(B_{\alpha \beta_{0}} ; G^{B, \alpha}\right)$.

Part (a). First, show that $\psi(Q)$ is not empty: by the block-synchronous strategy, we have $\mathcal{R}\left(a ; G^{A, \beta}\right) \times\{b\} \subset Q$ and also $\{a\} \times \mathcal{R}\left(b ; G^{B, \alpha}\right) \subset Q$, which implies $A_{\beta \alpha_{0}} \times B_{\alpha \beta_{0}} \in \psi(Q)$.

Part (b). To show that $\psi(Q)$ contains an attractor of $G_{m x}^{a}$, it suffices to show that $\psi(Q)$ contains all successors of its elements (in $G_{m x}^{a}$ ). So let $A_{u \alpha} \times B_{v \beta} \in \psi(Q)$. By definition of the mixed-mode asymptotic graph, its successors are of the form $A_{\beta \alpha_{1}} \times B_{v \beta}$ or $A_{u \alpha} \times$ $B_{\alpha \beta_{1}}$. But, if $\left(a_{0}, b_{0}\right) \in \pi\left(A_{u \alpha}\right) \times \pi\left(B_{v \beta}\right)$ then also $\left(a_{0}, b_{0}\right) \in Q$ and, from Part (a), we have $\mathcal{R}\left(a_{0} ; G^{A, \beta}\right) \times\left\{b_{0}\right\} \subset Q$ and also $\left\{a_{0}\right\} \times \mathcal{R}\left(b_{0} ; G^{B, \alpha}\right) \subset Q$. But this implies $A_{\beta \alpha_{1}} \times B_{v \beta} \in \psi(Q)$ and $A_{u \alpha} \times B_{\alpha \beta_{1}} \in \psi(Q)$, as wanted.

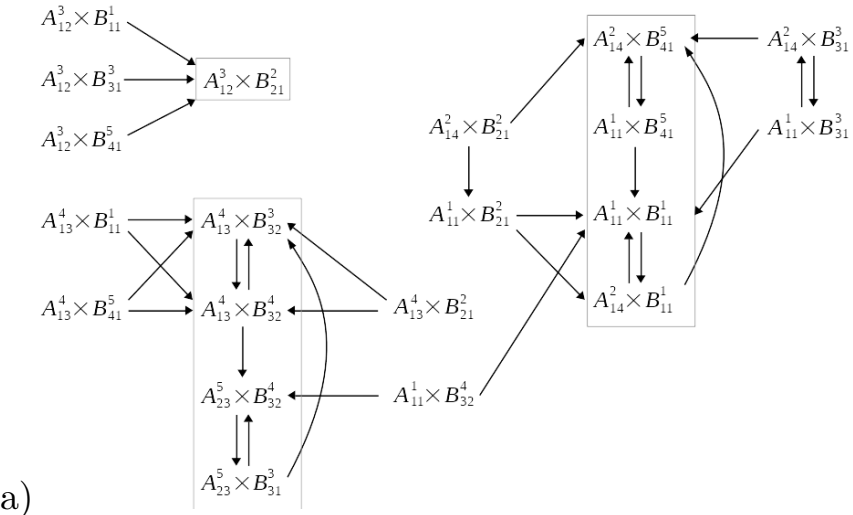

(a)

Figure 6: (a) The mixed updates asymptotic graph and (b) the full network state transition graph for the interconnection of modules $\Sigma_{A}$ and $\Sigma_{B}$ in (2).

Example Suppose that the two modules $\Sigma_{A}$ and $\Sigma_{B}$ given in (2) are both synchronously updated, but their interconnection is asynchronous. To construct the asymptotic graph $G_{m x}^{a}$, use the synchronous modules transition graphs $G^{X, u}$ shown in Fig. 2(b) and then construct the asymptotic graph using an asynchronous strategy. In this case, the sets of semi-attractors are the same as in the synchronous case (10), so that $\mathcal{V}$ has $5 \times 5=25$ vertices in $G_{m x}^{a}$. Fig. 6(a), The mixed strategy asymptotic graph has three attractors:

$$
\begin{aligned}
& Q_{m x}^{a, 1}=\left\{A_{12}^{3} \times B_{21}^{2}\right\}=\{0100\}, \\
& Q_{m x}^{a, 2}=\left\{A_{11}^{1} \times B_{11}^{1}, A_{11}^{1} \times B_{41}^{5}, A_{14}^{2} \times B_{11}^{1}, A_{14}^{2} \times B_{41}^{5}\right\}=\{0000,0010,1100,1110\} \\
& Q_{m x}^{a, 3}=\left\{A_{13}^{4} \times B_{31}^{3}, A_{13}^{4} \times B_{32}^{4}, A_{23}^{5} \times B_{31}^{3}, A_{23}^{5} \times B_{32}^{4}\right\}=\{1000,1010,1001,1011\} .
\end{aligned}
$$

However, Fig. 5(b) shows that the full network $G_{m x}^{I}$ has only two attractors, corresponding to $Q_{m x}^{a, 1}$ and $Q_{m x}^{a, 3}$. It follows that $Q_{m x}^{a, 2}$ is a spurious attractor, which does not correspond to any true attractor of $G_{m x}^{I}$. To understand the origin of this spurious result, compare the edges linking the following equivalent vertices in $G_{m x}^{I}$ and $G_{m x}^{a}$ :

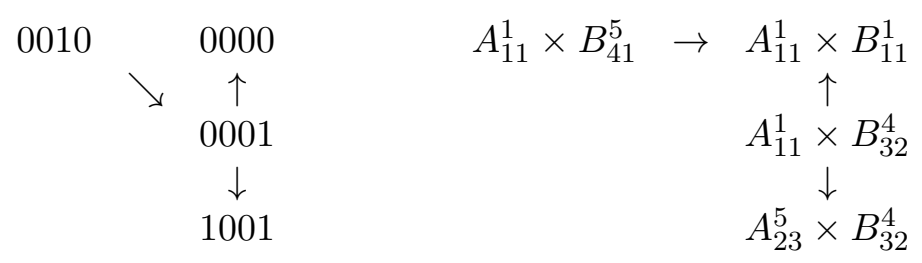


and note that the edge $A_{11}^{1} \times B_{41}^{5} \rightarrow A_{11}^{1} \times B_{32}^{4}$ is not allowed in $G_{m x}^{a}$, by definition (see edge rule (5)). Note also that $0010 \rightarrow 0001 \rightarrow 1001$ is the only pathway in $G_{m x}^{I}$ that allows transitions from the states in set $Q_{m x}^{a, 2}$ to those in set $Q_{m x}^{a, 3}$. The definition of the edges of $G_{m x}^{a}$ together with the lack of redundancy among pathways in $G_{m x}^{I}$ originates a spurious attractor in $G_{m x}^{a}$.

As before, this result can be extended to the interconnection between 3 or more synchronous blocks. Furthermore, if the strategy among blocks is asynchronous, it is clear that each block could follow any updating strategy, since only its state transition graphs $G^{X, u}$, $u \in\left\{1,2, \ldots, 2^{p_{X}}\right\}$, are needed to construct the asymptotic graph.

\section{The synchronous vs. asynchronous dynamics dichotomy}

The different dynamics induced by synchronous or asynchronous updating strategies is well illustrated through the construction of the asymptotic graphs.

In practice, we can compute all or most of the attractors of the interconnected network using only the knowlegde of the two individual modules, through the computation of a new graph of (much) reduced dimension, the asymptotic graph. Each strategy - either updating all variables simultaneouly or only one at a time - leads to a different construction for the asymptotic graph and to two apparently opposite results:

$$
\mathcal{A}\left(G^{I}\right) \supseteq \mathcal{A}\left(G^{a}\right), \quad \mathcal{A}\left(G_{m x}^{I}\right) \subseteq \mathcal{A}\left(G_{m x}^{a}\right), \quad \mathcal{A}\left(G_{*}^{I}\right) \subseteq \mathcal{A}\left(G_{*}^{a}\right) .
$$

In asynchronously interconnected networks, it appears that the information on the asymptotic behavior is completely included in the state transition graphs of the two separate modules, and this for any possible pair of modules. The asymptotic graph scheme may sometimes introduce a false attractor. Roughly speaking, the asynchronous nature of the dynamics is reminiscent of a slow/fast timescale separation, since each part of an attractor (either the A or B part) is fixed while the other part evolves rapidly inside its own module: the dynamics within each module is "fast" when compared to the timescale of the interconnection. Thus, the asynchronous asymptotic graph contains one representative of each family of pathways leading to any given attractor. In some particular cases, the families of pathways are not adequately represented in $G_{*}^{a}$, thereby leading to the appearance of a spurious attractor as illustrated by the example in Section 5.

In contrast, in synchronously interconnected networks, by construction the asymptotic graph contains actual pathways of $G^{I}$ (those starting from an initial choice of vertices, $\mathcal{W}_{0}$ ), so it cannot introduce any spurious attractors. In synchronous networks, each state has a unique successor and converges to a single attractor. The basins of attraction are pairwise disjoint and deterministically associated with a unique attractor. So, in general, the attractors associated to a pair of modules $\Sigma_{A}, \Sigma_{B}$ for $\Sigma_{I}$ may not sample appropriately all the basins of attraction, hence some attractors of $G^{I}$ will be "forgotten" in $G^{a}$. This is the case even in straigthforward networks such as the positive loop of size $n \geq 4$.

This difference in the structure of asynchronous and synchronous networks joins the work of Demongeot and colleagues [13] who have analysed the basins of attraction of synchronous networks, their sizes and the distances between them, as indicators of robustness. A small distance between two basins of attraction means that perturbations to an initial configuration are more likely to induce a switch from one basin to another, and hence to change the asymptotic behavior of the network.

The main disadvantage of the asymptotic graph construction is the possibility of generating spurious attractors in the case of asynchronous updates, or the failure to recover all attractors 

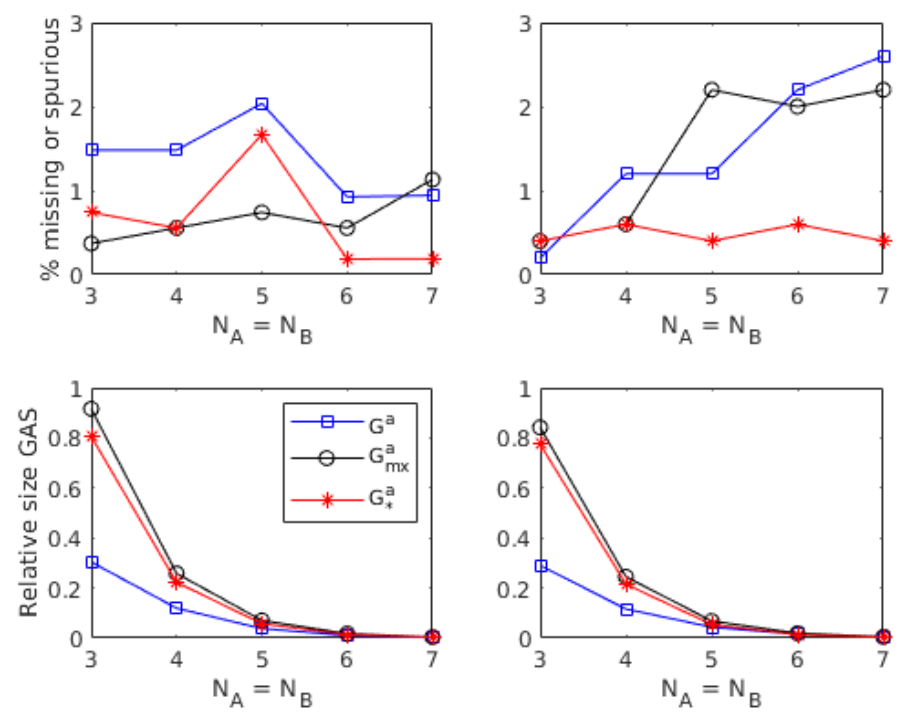

Figure 7: Performance of the asymptotic graph method for synchronous (blue curves), mixed (black curves), or asynchronous (red curves) interconnected networks. We considered two classes of networks: Horn Boolean functions (left column) and unate cascade functions (right column). For each class and each $N_{A}, 500$ networks were generated, formed by a pair of modules of equal size $N_{A}=N_{B} \in\{3,4,5,6,7\}$ and $N_{i n}=2$. For the three strategies, the corresponding full graphs and asymptotic graphs were computed. The plots compare percentage of failures (top) and the sizes of the asymptotic graph relative to the size of the full network, $\left|G^{a}\right| /\left|G^{I}\right|$ (bottom). 
under synchronous updates. However, our tests with randomly generated networks show that these errors are very small and decrease with size for networks with some structure (below $2 \%$, see Figure 7) and applications to biological networks modules has never shown any of these two "errors".

A quick comparison of the three stategies is shown in Figure 7. Two classes of Boolean functions were considered: unate cascade functions as characterized in [25], and Horn functions as characterized in [11], for $n$ variables:

$$
\begin{array}{ll}
\text { Unate cascades: } & f(x)=\hat{x}_{\sigma(1)} \diamond_{1}\left(\hat{x}_{\sigma(2)} \diamond_{2}\left(\ldots\left(\hat{x}_{\sigma(n-1)} \diamond_{n-1} \hat{x}_{\sigma(n)}\right) \ldots\right)\right), \\
\text { Horn functions: } & f(x)=\bigvee_{i=1}^{m}\left(\bigwedge_{j \in P_{i}} x_{j} \bigwedge_{k \in Q_{i}} \neg x_{k}\right)
\end{array}
$$

where $\sigma$ is a permutation of $\{1, \ldots, n\}, \hat{x}$ is either $x$ or $\neg x$, and $\diamond_{i}$ represents either the logical AND or the logical OR; for Horn functions $1 \leq m \leq 6,0 \leq\left|P_{i}\right| \leq n$ and $0 \leq\left|Q_{i}\right| \leq 1$, that is, each term in a Horn function has at most one negated variable. To generate Horn functions, the integers $m,\left|P_{i}\right|$, and $\left|Q_{i}\right|$ were randomly chosen out of a uniform distribution in the given intervals. To obtain unate cascade functions, a permutation and a sequence of $n-1$ logical operations were randomly generated from uniform distributions. For the computation of each of the three asymptotic graphs for each class, A total of $500 \times 5$ networks were randomly generated as pairs of modules with $N_{A}=N_{B} \in\{3,4,5,6,7\}$ and $N_{i n}=2$, for each class (each symbol corresponds to an average over 500 networks). For each of these networks, we computed the three asymptotic graphs and the full interconnected graph and compared the attractors. It is immediate to observe that the asynchronous strategy gives the most accurate results, with the lowest percentage of errors.

For the analysis of large networks, it is clear that synchronous updates have a lower computational cost. Thus, in view of Section 5, the most advantageous solution is to use a mixed strategy for analysis of interconnections: consider synchronous individual modules and then construct an asynchronous asymptotic graph. In cases where each individual module has its own time-scale and their communication happens at a slower pace, mixed strategies are a good option.

\section{Composition of biological networks by feedback interconnec- tion}

A recurrent problem in biology is to study the emergent dynamics arising from the coupling of two or more different phenomena $[22,4]$. Although many biological phenomena are currently represented by mathematical models, there are no specific tools to systematically explore the interconnections between two existing models. Our asymptotic graph method addresses this problem by providing a framework to analyse the joint dynamics of two known networks. Given two or more previously developed models, our method requires only an investigation of the input-output connections between the two networks (in Fig. 1, finding $u=h_{B}(b)$ and $\left.v=h_{A}(a)\right)$.

To illustrate our method, four pairs of Boolean networks that model distinct phenomena in several organisms have been coupled to analyze the corresponding joint dynamics. These pairs are listed in Table 2 by order of size/complexity. (For a complete description and construction of the models, please refer to the bibliography cited in the Table.)

Following the discussion on Section 6, we will consider synchronous modules and then compare the synchronous and mixed asymptotic graphs. 
Table 2: Composition of pairs of Boolean networks. The size of each module is given in parentheses $\left(N_{A}\right.$ and $N_{B}$ state the number of variables). The last column lists the references where the original Boolean networks are constructed.

\begin{tabular}{lccc} 
Organism & System A $\left(N_{A}\right)$ & System B $\left(N_{B}\right)$ & References \\
\hline \hline Cyanobacteria & Clock $(5)$ & TTC $(3)$ & {$[7],[16]$} \\
\hline Mammalians & Cell cycle $(10)$ & Clock $(7)$ & {$[17],[10]$} \\
\hline Vertebrates & T helper A (10) & T helper B $(13)$ & {$[30]$} \\
\hline Arabidopsis thaliana & Cell cycle (14) & GHRN (16) & {$[32],[20]$} \\
\hline
\end{tabular}

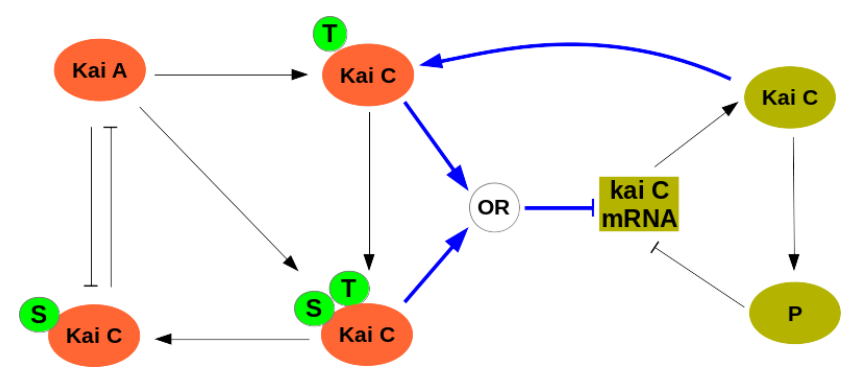

Figure 8: The interconnected cyanobacteria clock (left) and transcription/translation cycle (right). Bold blue arrows denote input/output connections. The protein KaiA affects KaiC-S $\left(C_{S}\right)$ at a different rate than KaiC-T $\left(C_{T}\right)$ and KaiC-TS $\left(C_{T S}\right)$. Therefore, KaiA is represented by two variables, $A_{1}$ (low KaiA level) and $A_{2}$ (high KaiA level). The unphosphorylated protein Kai $\mathrm{C}$ is denoted by $C_{U}$.

The notation used throughout these examples is the same as before, using the lexicographical order for Boolean numbers: $A_{25}^{3}$ means that this semi-attractor belongs to the graph $G^{A, 2}$, it has input "2" (Boolean 001) and output "5" (Boolean 100). The superscript "3" means that it is semi-attractor number 3 in the total count of semi-attractors of module A.

\subsection{The cyanobacteria example}

Circadian rhythms in cyanobacteria are widely studied and the corresponding mechanism is very well known: it is characterized by an ordered sequence of phosphorylations in the protein Kai $\mathrm{C}$ under the regulation of another protein, Kai A. This mechanism can be faithfully reproduced in vitro (see [35] and references therein). In living cyanobacteria, the clock mechanism interacts with the transcription/translation cycle (TTC) [16].

The two cyanobacteria modules The Boolean clock mechanism $\left(\Sigma_{A}\right)$ was developed in [7] and a simple TTC circuit is composed by unphosphorylated protein Kaic $\mathrm{C}\left(C_{U}\right)$ and its mRNA $(R)$, and a "lumped" protein $P$ closes the circuit:

$$
\begin{aligned}
& A_{1}^{+}=\neg C_{S} \vee A_{2} \\
& A_{2}^{+}=\neg C_{S} \wedge A_{2} \quad R^{+}=\neg v \wedge \neg P \\
& \Sigma_{A}: C_{T}^{+}=u \wedge A_{1} \\
& C_{T S}^{+}=A_{1} \wedge C_{T} \\
& C_{S}^{+}=\neg A_{2} \wedge C_{T S} \\
& \Sigma_{B}: C_{U}^{+}=R \\
& P^{+}=C_{U}
\end{aligned}
$$

Based on [16], the interactions are as follows:

$$
u=h_{B}(b)=C_{U}, \quad v=h_{A}(a)=C_{T} \vee C_{T S} .
$$


Computation of the state transition graphs for each module shows that there are five semiattractors for each module, as follows:

$G^{A, 1}: \quad A_{11}^{1}=\{10000\}, \quad A_{11}^{2}=\{11000\}$,

$G^{A, 2}: \quad A_{21}^{3}=\{00000,00001,10000\}, \quad A_{22}^{4}=\{00111,10100,10110,10111\}, \quad A_{22}^{5}=\{111(0\})$

and

$$
\begin{array}{ll}
G^{B, 1}: & B_{11}^{1}=\{000,001,100\}, \quad B_{12}^{2}=\{011,110,111\}, \quad B_{11}^{3}=\{101\}, \quad B_{12}^{4}=\{010\}, \\
G^{B, 2}: & B_{21}^{5}=\{000\},
\end{array}
$$

Note that $A_{21}^{3} \cup A_{22}^{4}$ form an attractor of $\Sigma_{A}$ (under input 2), which corresponds to the circadian clock oscillatory orbit:

$$
00000 \rightarrow 10000 \rightarrow 10100 \rightarrow 10110 \rightarrow 10111 \rightarrow 00111 \rightarrow 00001 \rightarrow 00000
$$

where the Kai C protein is sequencially phosphorylated on its site $T$ (10100), then $T$ and $S$ (10110), then only on site $S$ (10111), which inhibits Kai A (00111), leading to repeat the cycle. Similarly, the attractor $B_{11}^{1} \cup B_{12}^{2}$ represents the full TTC cycle with Kai C mRNA production (100) followed by protein production (110), which induces the full pathway (111) and leads to cycle repetition.

The cyanobacteria synchronous asymptotic graph Computation of the synchronous asymptotic graph leads to the transition graph shown in Fig. 11. This graph contains 52 states (instead of the $2^{8}=256$ of the full interconnected system), represented by products of semi-attractors or states $a \times b$ (i.e., Boolean states $a$ of $\Sigma_{A}$ and $b$ of $\Sigma_{B}$ ) and two cyclic attractors:

$$
\begin{array}{ll}
Q^{a, 1}: \quad 00000 \times 100 \rightarrow 10000 \times 110 \rightarrow 10100 \times 111 \rightarrow 10110 \times 011 \rightarrow 10111 \times 001 \rightarrow \\
& \rightarrow 00011 \times 000 \rightarrow 00001 \times 000 \rightarrow \cdots \\
Q^{a, 2}: \quad & 11000 \times 100 \rightarrow 11000 \times 110 \rightarrow 11100 \times 111 \rightarrow 11110 \times 011 \rightarrow 11110 \times 001 \rightarrow \\
& \rightarrow 11010 \times 000 \rightarrow 11000 \times 000 \rightarrow \cdots .
\end{array}
$$

The first attractor oscillates simultaneaously through the clock and TTC orbits (except that 00111 is replaced by 00011, an equivalent state at this step), showing that the interconnected system has synchronized clock and transcription/translation oscillations, as expected for wild type cells.

The second attractor still cycles through TTC but the clock evolves among states which contain a high concentration of protein Kai A $\left(A_{11}^{2}\right.$ and $\left.A_{22}^{5}\right)$, thus preventing the formation of $C_{S}$ and subsequent re-initialization of the Kai proteins phosphorylation cycle. Such dynamics can indeed be observed experimentally [35]: a high expression of Kai A prevents circadian clock oscillations.

The cyanobacteria mixed mode asymptotic graph To compare updating strategies, the mixed mode asymptotic graph is shown in Fig. 12. It is composed of 25 states (only products of semi-attractors) and also contains two attractors:

$$
\begin{aligned}
& Q_{m x}^{a, 1}=\left\{A_{11}^{1} \times B_{11}^{1}, A_{11}^{1} \times B_{12}^{2}, A_{11}^{1} \times B_{21}^{5}, A_{21}^{3} \times B_{12}^{2}, A_{21}^{3} \times B_{11}^{1}, A_{22}^{4} \times B_{12}^{2}, A_{22}^{4} \times B_{21}^{5}\right\} \\
& Q_{m x}^{a, 2}=\left\{A_{22}^{5} \times B_{12}^{2}, A_{22}^{5} \times B_{21}^{5}, A_{11}^{2} \times B_{12}^{2}, A_{11}^{2} \times B_{21}^{5}, A_{11}^{2} \times B_{11}^{1}\right\} .
\end{aligned}
$$




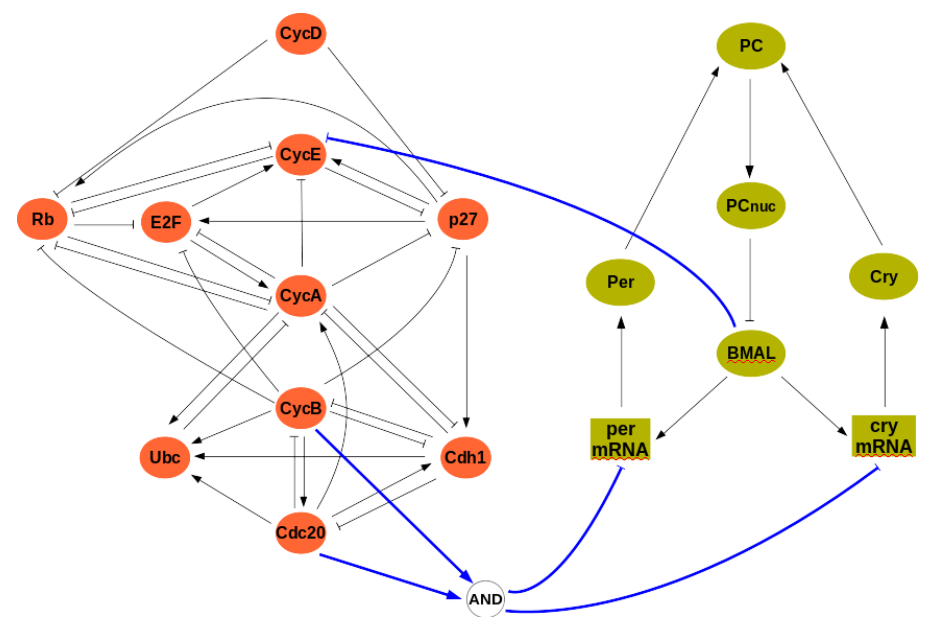

Figure 9: The interconnected mammalian cell cycle (left, adapted from [17]) and clock (right, adapted from [10]). Bold blue arrows denote input/output connections.

From equations (11) and (12), observe that attractors $Q_{m x}^{a, i}$ correspond to attractors $Q^{a, i}$. Merging semi-attractors that belong to the same attractor, $Q_{m x}^{a, 1}$ has the following compact dynamics:

$$
\begin{array}{cccc}
A_{22}^{4} \times B_{21}^{5} & \leftarrow & A_{2 .}^{3} \times B_{1}^{1} \\
\downarrow & & \uparrow \downarrow \\
A_{11}^{1} \times B_{21}^{5} & \rightarrow & A_{11}^{1} \times B_{1}^{1} .
\end{array}
$$

meaning that there is (as in the synchronous asymptotic graph) a simultaneous clock and TTC oscillation $\left(A_{2}^{3} \times B_{1}^{1}\right.$. $)$, but also the possibility that the clock oscillator still follows a clear sequence with Kai $\mathrm{C}$ successively phosphorylated in its three possible combinations $\left(A_{22}^{4}\right)$ and then all unphosphorylated $A_{11}^{1}$, while the TTC is arrested (fixed at $B_{21}^{5}$ ). This last case may represent a situation where protein production is slower or has a higher period than the clock.

\subsection{Mammalian example}

The interactions between the mammalian cell cycle and circadian clock are currently generating a large amount of interest in biology, as the progress in single cell measurements allows answering specific questions [18]. These new experiments are uncovering unexpected bi-directional links between the two modules. 
The two mammalian modules The cell cycle Boolean model (module $\Sigma_{A}$ ) is taken from [17] and has the following variables:

$$
\begin{aligned}
C y c D^{+} & =C y c D \\
R b^{+} & =(\neg C y c D \wedge \neg C y c E \wedge \neg C y c A \wedge \neg C y c B) \vee(p 27 \wedge \neg C y c D \wedge \neg C y c B) \\
E 2 F^{+} & =(\neg R b \wedge \neg C y c A \wedge \neg C y c B) \vee(p 27 \wedge \neg R b \wedge \neg C y c B) \\
C y c E^{+} & =\neg u \wedge(E 2 F \wedge \neg R b) \\
C y c A^{+} & =(E 2 F \wedge \neg R b \wedge \neg C d c 20 \wedge \neg(C d h 1 \wedge U b c)) \vee(C y c A \wedge \neg R b \wedge \neg C d c 20 \wedge \neg(C d h 1 \wedge U b c)) \\
p 27^{+} & =\neg C y c D \wedge \neg C y c E \wedge \neg C y c A \wedge \neg C y c B \\
C d c 20^{+} & =C y c B \\
C d h 1^{+} & =(\neg C y c A \wedge \neg C y c B) \vee C d c 20 \vee(p 27 \wedge \neg C y c B) \\
U b c^{+} & =\neg C d h 1 \vee(C d h 1 \wedge U b c \wedge(C d c 20 \vee C y c A \vee C y c B)) \\
C y c B^{+} & =\neg C d c 20 \wedge \neg C d h 1,
\end{aligned}
$$

as described in [17], where $C y c X$ represent the four cyclins involved in the cell cycle. The clock model (module $\Sigma_{B}$ ) is based on [10]:

$$
\begin{aligned}
B M A L^{+} & =\neg P C n u c \\
m P E R^{+} & =\neg v \wedge B M A L \\
m C R Y^{+} & =\neg v \wedge B M A L \\
p P E R^{+} & =m P E R \\
p C R Y^{+} & =m C R Y \\
P C^{+} & =p P E R \wedge p C R Y \\
P C n u c^{+} & =P C .
\end{aligned}
$$

In the clock model, $m X$ and $p X$ denotes mRNA and protein corresponding to gene $X$, while $P C$ denotes the complex formed by the proteins PER and CRY, and PCnuc denotes this complex in the nucleus.

Testing interactions between modules Although there are currently many questions on the form of interaction between mammalian cell cycle and circadian clock [19], it seems clear that clock's $B M A L$ acts on the cell cycle, possibly at different stages. Conversely, no conclusive biological knowledge exists on how the cell cycle may affect the clock. To include a basic link, we have considered that during mitosis all gene expression is arrested (in this model, mitosis can be modeled as $C d c 20 \wedge C y c B$ ). The interconnection between modules is thus given by (see Fig. 9):

$$
u=h_{B}(b)=B M A L, \quad v=h_{A}(a)=C d c 20 \wedge C y c B .
$$

$B M A L$ acts negatively during $\mathrm{G} 1, \mathrm{~S}$ or M-phases of the cell cycle. We analyzed three different interconnection models, one for each of these possibilities, and compared the results (see Table 4). The three cases give similar results in terms of joint behavior. For a closer analysis consider, for instance, $B M A L$ acting during G1 (here represented by a negative efect on cyclin 
E). In this case, the attractors of each module are:

$$
\begin{aligned}
G^{A, 1}: \quad A_{11}^{1} & =\{0100010100\}, \\
A_{11}^{2} & =\{1000001110,1000100011,1001100000,1010000110,1011000100,1011100100\}, \\
A_{12}^{3} & =\{1000101011\}, \\
G^{A, 2}: \quad & A_{21}^{4}=\{0100010100\}, \\
A_{21}^{5} & =\{1000001110,1000100000,1000100011,1010000100,1010000110,1010100100\}, \\
A_{22}^{6} & =\{1000101011\}, \\
G^{B, 1}: \quad & B_{11}^{1}=\{0000000,0000001,0000011,0001111,0111111\}, \\
B_{12}^{2} & =\{1000000,1110000,1111100,1111110,1111111\}, \\
B_{11}^{3} & =\{0000010,0001101,0110000,0110011,0111110\}, \\
B_{12}^{4} & =\{1000001,1001100,1001111,1110010,1111101\}, \\
B_{11}^{5} & =\{0001100,0001110,0110001,0111100,0111101\}, \\
B_{12}^{6} & =\{1000010,1000011,1001110,1110001,1110011\}, \\
B_{11}^{7} & =\{0110010\}, B_{12}^{8}=\{1001101\}, \\
B_{22}^{9} & =\{1000000\}
\end{aligned}
$$

As expected, the attractors of the graph $G^{A, 1}$ correspond exactly to those listed in [17]. Attractors $A_{11}^{1}$ and $A_{21}^{4}$ correspond to the quiescent cell state while the (full) attractor $A_{11}^{2} \cup A_{12}^{3}$ corresponds to the known cell cycle progression. A similar cycle is also recovered in $G^{A, 2}$, with the difference that states 1001100000, 1011000100, and 1011100100 are replaced, respectively, by 1000100000, 1010000100, and 1010100100: the Boolean sequence of the latter three equals that of the former three except in the 4 th digit, which is set to 0 . This is because $u=2$ implies $C y c E^{+} \equiv 0$ (and $C y c E$ is the 4 th variable). In both cases, the semi-attractors $A_{12}^{3}$ and $A_{22}^{6}$ represent mitosis.

The clock mechanism admits a single steady state $\left(B_{22}^{9}\right)$ in the case $v=2$, which corresponds to mitosis. This makes sense, as all gene expression is arrested. Outside mitosis (case $v=1)$, there are four different oscillatory cycles $\left(B_{11}^{1} \cup B_{12}^{2}, B_{11}^{3} \cup B_{12}^{4}, B_{11}^{5} \cup B_{12}^{6}\right.$, and $\left.B_{11}^{7} \cup B_{12}^{8}\right)$ where only the first two correspond to circadian oscillations.

Mammalian synchronous and mixed mode asymptotic graphs Among the different interaction schemes tested, all allow at least one cycle that captures joint oscillations of cell cycle and clock. For the scheme where BMAL affects $C y c E$ (13), the synchronous asymptotic graph has five attractors, but only one of these recovers joint oscillations $\left(Q^{2}\right)$, all others corresponding to a quiescent cell with oscillatory clock:

$$
\begin{aligned}
Q^{a, 1}= & A_{21}^{4} \times\left(B_{11}^{7} \cup B_{12}^{8}\right) \\
Q^{a, 2}= & \{1000100000 \times 1110001,1000100011 \times 0111100,1000101011 \times 1001110,1000001110 \times 1000011, \\
& 1010000110 \times 0110001,1011000100 \times 0001100,1011100100 \times 1000010\} \\
Q^{a, 3}= & \left(A_{11}^{1} \cup A_{21}^{4}\right) \times\left(B_{11}^{3} \cup B_{12}^{4}\right) \\
Q^{a, 4}= & \left(A_{11}^{1} \cup A_{21}^{4}\right) \times\left(B_{11}^{1} \backslash\{0000000\} \cup B_{11}^{5} \cup B_{22}^{9} \cup B_{12}^{2}\right) \\
Q^{a, 5}= & \left(A_{11}^{1} \cup A_{21}^{4}\right) \times\left(B_{11}^{5} \cup B_{12}^{6}\right)
\end{aligned}
$$

Attractor $Q^{a, 2}$ represents a joint oscillatory solution taking specific pairs $(a, b)$ with $a \in A_{11}^{2} \cup$ $A_{12}^{3}$ or $a \in A_{21}^{5} \cup A_{22}^{6}$ and $b \in B_{11}^{5} \cup B_{12}^{6}$. 
The mixed mode graph has also five attractors, which correspond to each of $Q^{a, i}$. In fact, we can see from Fig. 14 that there is set equality $Q^{a, i}=Q_{m x}^{a, i}$ for $i=1,3,4,5$ but strictly inequality for $Q^{a, 2} \subset Q_{m x}^{a, 2}$.

The attractors $Q^{a, i} i=1,3,4,5$ represent quiescent cells with ongoing circadian oscillations (as observed in hair cells [33]), while $Q^{a, 2}$ represents the joint cell division cycle and clock oscillatory behavior. The mixed mode asymptotic graph shows how this joint behavior emerges from the combination of the module's periodic orbits (see left attractor in Fig. 14). Merging semi-attractors that belong to the same attractor, $Q_{m x}^{a, 2}$ can be represented in the compact form:

$$
\begin{array}{cccc}
A_{12}^{3} \times B_{22}^{9} & \leftarrow & A_{1 \cdot}^{2} \times B_{1}^{1} \\
\downarrow & & \uparrow \downarrow \\
A_{2 \cdot}^{5} \times B_{22}^{9} & \leftrightarrows & A_{2 \cdot}^{5} \times B_{1}^{1}
\end{array}
$$

where $A_{1}^{2}$. $=A_{11}^{2} \cup A_{12}^{3}, A_{2}^{5}=A_{21}^{5} \cup A_{22}^{6}$, and $B_{1}^{1}=B_{11}^{1} \cup B_{12}^{2}$. The cell cycle and clock may jointly oscillate and alternate states with a regular cycle of cyclin $\mathrm{E}$ (present mostly through $\mathrm{S}$ phase and mitosis) or eventually switch to a joint cycle with absence of cyclin E. However, at mitosis $\left(A_{12}^{3}\right)$, the clock may switch to its steady state $\left(B_{22}^{9}\right)$, which leads directly to a full degradation of cyclin $\mathrm{E}$ in the cell cycle $\left(A_{2}^{5}\right.$.).

\subsection{T helper cells regulatory network}

The third example analyzes the regulatory network developed in [30] to describe the differentiation of $\mathrm{T}$ helper cells, which are part of the vertebrate immune system, into one of three classes: Th0 precursor cells and Th1, Th2 effector cells. Network reconstruction is discussed in [30], it involves 19 variables and four external input variables, $X_{e} \in\{I F N \beta, I L 12, I L 18, T C R\}$. For the analysis in [30], these four inputs are all set to 0 but here, these external inputs are treated as constant variables, setting $X_{e}^{+}=X_{e}$ to allow for greater generality in the results. Thus, the 23 variable network was first split into two modules, of 10 and 13 variables, respectively:

$$
\begin{aligned}
& G A T A 3^{+}=(\text {GATA3 } \vee S T A T 6) \wedge \neg \text { Tbet } \\
& I F N \beta^{+}=I F N \beta \\
& I F N \beta R^{+}=I F N \beta \\
& I L 4^{+}=G A T A 3 \wedge \neg S T A T 1 \\
& \Sigma_{A}: \quad I L 4 R^{+}=I L 4 \wedge \neg S O C S 1 \\
& J A K 1^{+}=u \wedge \neg S O C S 1 \\
& S O C S 1^{+}=S T A T 1 \vee \text { Tbet } \\
& S T A T 1^{+}=I F N \beta R \vee J A K 1 \\
& S T A T 6^{+}=I L 4 R \\
& \text { Tbet }^{+}=(\text {STAT } 1 \vee \text { Tbet }) \wedge \neg G A T A 3
\end{aligned}
$$




$$
\begin{aligned}
I F N \gamma^{+} & =\left(I R A K \vee N F A T \vee S T A T 4 \vee v_{3}\right) \wedge \neg S T A T 3 \\
I F N \gamma R^{+} & =I F N \gamma \\
I L 10^{+} & =v_{1} \\
I L 10 R^{+} & =I L 10 \\
I L 12^{+} & =I L 12 \\
I L 12 R^{+} & =I L 12 \\
I L 18^{+} & =I L 18 \\
I L 18 R^{+} & =I L 18 \wedge \neg v_{2} \\
I R A K^{+} & =I L 18 R \\
\Sigma_{B}: \quad & =I C R \\
S T A T^{+} & =I L 10 R \\
S T A T 3^{+} & =I L 12 R \wedge \neg v_{1} \\
T C R^{+} & =T C R
\end{aligned}
$$

where the interconnection between modules is given by:

$$
u=h_{B}(b)=I F N \gamma R, \quad v=h_{A}(a)=(\text { GATA3, STAT6, Tbet })^{\prime} .
$$

Module $A$ is single input and has the following 12 semi-attractors:

$$
\begin{array}{ll}
G^{A, 1}: & A_{11}^{1}=\{0000000000\}, A_{12}^{2}=\{0000001001\}, A_{12}^{3}=\{0110001101\}, A_{17}^{4}=\{1001100010\}, \\
A_{15}^{5}=\{1110001100\}, & \\
G^{A, 2}: & A_{22}^{6}=\{0000001001\}, A_{22}^{7}=\{0110001101\}, \\
A_{25}^{8} & =\{1000001000,1001000000,1001110000\}, A_{27}^{9}=\{1000001110,1000111110,1001110110\}, \\
A_{25}^{10} & =\{1000111000\}, A_{27}^{11}=\{1001000110\}, A_{25}^{12}=\{1110001100\} .
\end{array}
$$

Module $B$ has 3 inputs, and therefore eight fixed input graphs $G^{B, v}$, with altogether 64 single state attractors (not listed here).

For the interconnection of the two systems, the synchronous and mixed asymptotic graphs give similar results, both with 33 single state attractors, which can be divided into the following five groups:

$$
\begin{aligned}
& A_{11}^{1} \times B_{11}^{1}=\{0000000000\} \times\{0000000000000\} \\
& A_{17}^{4} \times\{0011 * * * 00 * 10 *\}, \quad A_{15}^{5} \times\{0011 * * * * * * 10 *\}, \\
& A_{22}^{6} \times\{1100 * * * * * * 0 * *\}, \quad A_{22}^{7} \times\{1100 * * * * * * 0 * *\},
\end{aligned}
$$

where the symbol $*$ indicates that the corresponding digit may equal either 0 or 1 . In the particular case where all the external input variables are set to $0, X_{e} \equiv 0$, there are only 3 attractors for the full $\mathrm{T}$ helper cell regulatory network, as expected. Indeed, this was the only case considered in [30], which lists the three attractors corresponding to the three $\mathrm{T}$ helper cell phenotypes, Th0, Th1, and Th2.

Our analysis was more general, since the four external input variables $\left(X_{e}\right)$ were allowed to take all possible combinations of 0,1 values, leading to a total of 33 distinct attractors for the $\mathrm{T}$ helper cell network. However, notice that these attractors follow some specific patterns, and five distinct groups can be identified, according to the cytokines expressed or not in each attractor. Furthermore, each group of attractors can then be classified as a representative of a distinct $\mathrm{T}$ helper cell phenotype, as a function of 10 cytokines and proteins that typically discriminate between the phenotypes.

As summarized in [30], precursor T helper cells, or Th0, do not produce any of the cytokines represented in this model. The interconnected network $\left(\Sigma_{A}, \Sigma_{B}\right)$ describes the differentiation 
into the two types of effector cells. Th1 type cells are characterized by high expression of cytokine $I F N \gamma$, protein $S O C S 1$, and transcription factor Tbet. In contrast, the dominant characteristic of Th2 cells is a high expression of cytokine $I L 4$ and its receptor $I L 4 R$. It is known that SOCS1 inhibits the IL4 pathway, hence SOCS1 is hardly present in Th2 cells. Moreover, it appears that GATA3 forms a positive feedback loop with $I L 4$, hence it is also expressed in Th2 cells. Another cytokine, IL10, is also observed in Th2 cells: its expression is enhanced by $I L 4$ and depends on the presence of STAT3.

Table 3: Classification of attractors into T helper cell phenotypes.

\begin{tabular}{|c|c|c|c|c|c|}
\hline Variable & $A_{11}^{1} \times B_{11}^{1}$ & $\begin{array}{c}A_{17}^{4} \times \\
\{0011 \cdots 00 \cdot 10 \cdot\}\end{array}$ & $\begin{array}{c}A_{15}^{5} \times \\
\{0011 \cdots \cdots 10 \cdot\}\end{array}$ & $\begin{array}{c}A_{22}^{6} \times \\
\{1100 \cdots \cdots \cdot \cdots \cdot\}\end{array}$ & $\begin{array}{c}A_{22}^{7} \times \\
\{1100 \cdots \cdots \cdot \cdots\}\end{array}$ \\
\hline$I F N \gamma$ & 0 & 0 & 0 & 1 & 1 \\
\hline$I F N \gamma R$ & 0 & 0 & 0 & 1 & 1 \\
\hline$I L 10$ & 0 & 1 & 1 & 0 & 0 \\
\hline$I L 10 R$ & 0 & 1 & 1 & 0 & 0 \\
\hline$I L 4$ & 0 & 1 & 0 & 0 & 0 \\
\hline$I L 4 R$ & 0 & 1 & 0 & 0 & 0 \\
\hline$S T A T 3$ & 0 & 1 & 1 & 0 & 0 \\
\hline GATA3 & 0 & 1 & 1 & 0 & 0 \\
\hline SOCS1 & 0 & 0 & 1 & 1 & 1 \\
\hline Tbet & 0 & 0 & 0 & 1 & 1 \\
\hline$I F N \beta$ & 0 & 0 & 1 & 0 & 1 \\
\hline Cell type & Th0 & Th2 & Th1p & Th1 & Th1 \\
\hline
\end{tabular}

Indeed, all the cytokines and proteins that distinguish between effector cell types are constant in each of the five attractor families, as seen in Table 3. Concerning the external inputs, only $I F N \beta$ has constant expression in each group.

It is clear that $A_{11}^{1} \times B_{11}^{1}$ represents precursor cells, Th0, with no expressed variables. There are two attractor groups (columns five and six) that correspond to Th1 phenotype, with all the required proteins expressed: $I F N \gamma, I F N \gamma, S O C S 1$, and Tbet. There is one group (column three) which exactly corresponds to phenotype Th2, as it expresses both $I L 4, I L 10$ and their receptors, as well as GATA3 and STAT3.

A fifth attractor group (column four) is identified, representing an intermediate type of cell which we classified as "pre-Th1" cell phenotype. While this group expresses most of Th2 characteristic proteins, it also expresses the Th1 protein SOCS1, which inhibits the dominant protein in Th2, IL4. Therefore, this group of attractors cannot be said to represent Th2. One of the differences between Th2 and Th1p is in the fact that Th1p is stimulated by external input $I F N \beta$. Experimental evidence [3] shows that IFN $\beta$ promotes Th1 cell maturation, by increasing $I F N \gamma$ production.

The T helper cell network does reproduce this fact, as shown in Fig. 10: since both GATA3 and Tbet have self-loops, it suffices to apply a perturbation (to Tbet for instance) to invert the expression levels of GATA3 and Tbet, which will in turn lead to the expression of the other proteins in a Th1 phenotype. Conversely, it can also be remarked that suppression of external input IFN $\beta$ will lead to loss of SOCS1 expression and subsequent de-repression of 
$I L 4 R$, which would determine a Th2 phenotype.

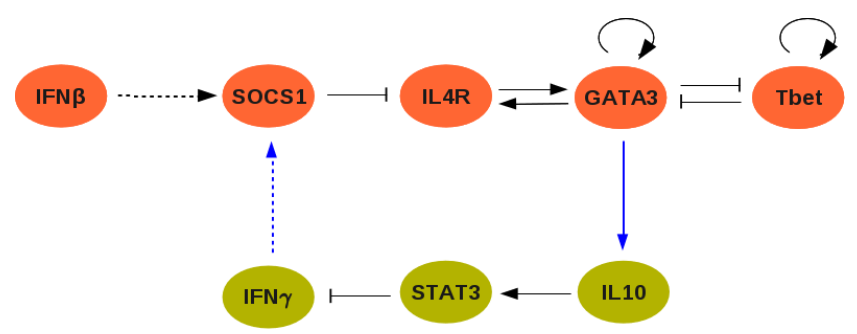

Figure 10: Partial visualization of the $\mathrm{T}$ helper cell network, containing only the nodes and edges involved in maturation of Th1p towards Th1 or Th2, as discussed in Section 7.3. $\Sigma_{A}$ nodes are coloured red and $\Sigma_{B}$ nodes are coloured green. Dashed arrows denote a simplified pathway, composed of several steps.

As a final remark, it can be noted that Th1 phenotype exists both in the presence and absence of the external input $I F N \beta$. However, Th2 phenotype can only exist in the absence of $I F N \beta$, as expected from experimental evidence [3].

\subsection{Arabidopsis thaliana example}

In the last two years, two fundamental Arabidopsis thaliana signaling networks have been developed using Boolean models, one comprehensively representing the cell cycle [32] and another studying the formation of the root apical meristem (RAM) through a genetic-hormonal regulatory network (GHRN) [20]. Both have about 15 Boolean variables (we will not reproduce the Boolean networks here, as they are easily accessible through these references) the first has only one attractor while the later has 17 . Several points of possible interactions between these two networks are discussed in [20], notably two main actions of components of GHRN on the cell cycle: the first through auxin which modulates the transition from mitotic to endocycle in Arabidopsis, and the second through cytokinin which promotes the expression of an APC activator in the root meristem. In the GHRN1 model, auxin is represented by WOX5 (transcription factor Wuschel-related-homeobox-5) and cytokinin by ARR2 (Arabidopsis Response Regulator 2) . The action of the cell cycle on the GHRN was again represented by arresting all gene expression during mitosis, here modeled by $A P C \wedge C y c B$.

The interconnection between modules is thus given by:

$$
u=h_{B}(b)=[A R R 2, W O X 5]^{\prime}, \quad v=h_{A}(a)=A P C \wedge C y c B
$$

where $u$ inhibits expression of the five genes in GHRN1: SHORT-HYPOCOTYL2 (SHY2), SHORTROOT (SHR), SCARECROW (SCR), JACKDAW (JKD), and MAGPIE (MGP). Conversely, $v_{1}$ positively affects APC and $v_{2}$ negatively affects cyclin D.

As expected, the transition graphs $G^{A, 1}$ and $G^{B, 1}$ have exactly the same attractors obtained in $[32,20]$. The mixed mode asymptotic graph $G_{m x}^{a}$ has seven attractors, of the form:

$$
Q_{m x}^{a, k \ell}=\begin{gathered}
A_{11}^{1} \times B_{11}^{k} \\
\uparrow \downarrow \\
A_{12}^{2} \times B_{11}^{k}
\end{gathered} \rightarrow \begin{gathered}
A_{11}^{1} \times B_{21}^{\ell} \\
\uparrow \downarrow \\
A_{12}^{2} \times B_{21}^{\ell}
\end{gathered}, \quad Q_{m x}^{a, Q C}=A_{21}^{3} \times B_{12}^{6}
$$

where $A_{11}^{1} \cup A_{12}^{2}$ is the cell cycle attractor [32] and $A_{21}^{3}$ is an attractor representing quiescent cells, and satisfying $E 2 F a=1, S C F=C y c B=C y c A=0$. The attractors $B_{v \beta}^{\ell}$ represent 
some well known hormonal activity profiles, as listed in [20]:

$B_{12}^{6}$ : Quiescent center cells, QC

$B_{11}^{13}, B_{21}^{20}$ : $\quad$ Central pro-vascular tissues, in Meristem Transition Domain (C. Prov. TD3)

$B_{11}^{14}, B_{21}^{21}$ : $\quad$ Root Cap (R.C. 2)

$B_{11}^{15}, B_{21}^{22}$ : $\quad$ Cyclic attractor 2

$B_{11}^{16}$ : $\quad$ Central pro-vascular tissues, in Meristem Transition Domain (C. Prov. TD1)

$B_{11}^{17}$ : $\quad$ Root Cap (R.C. 1)

$B_{11}^{18}: \quad$ Cyclic attractor 1

$B_{11}^{23}$ : C. Prov. TD1, with SHY2=0

$B_{11}^{24}$ : $\quad$ R.C. 1 , with $\mathrm{SHY} 2=0$

$B_{11}^{25}$ : $\quad$ Cyclic attractor 1 , with $\mathrm{SHY} 2=0$

The mixed mode asymptotic graph thus predicts that the interconnected cell cycle / GHRN1 network will have seven possible types of asymptotic behavior, characterized by (14), where the pairs $(k, \ell) \in\{(13,20),(14,21),(15,22),(16,24),(17,25),(18,23)\}$. In summary, there is a quiescent cell steady state $A_{21}^{3} \times B_{12}^{6}$, where cell cycle is arrested. All other dynamical behaviors consist of a normal cell cycle together with a hormonal profile coresponding either to Root Cap formation or to central pro-vascular tissues, in the transition domain (TD) of the root apical meristem. The synchronous asymptotic graph obtains all attractors in (14) as well as some variations around the quiescent cell state $Q_{m x}^{a, Q C}$ (not shown).

To explore whether other dynamical behaviors may be obtained, a deep study of the interactions between the cell cycle and GHRN is needed, but this is out of the scope of our paper. Nevertheless, it stands out that our method constitutes a very practical tool to attack this question.

\section{Discussion and conclusions}

The state transition graph of a large network is a computationaly expensive object, especially for asynchronous networks. Likewise, computing the attractors of large Boolean networks is a NP-hard problem $[2,23]$. Here, we proposed a new method that computes the attractors of large Boolean networks which are constructed by assembling together sets of smaller, already known, modules, as is often the case in physics or biology, for instance.

Theoretical advantages The asymptotic graph for the feedback interconnection of two modules is a new object computed using only the state transition graphs and attractors of each of the modules. This is a much less demanding state transition graph to compute, as attested by our studies.

Other methods are very fast in computing all singletons for very large networks (up to $\approx 1000$ variables) [42], or very efficient in computing most types of attractors (up to $\approx 100$ under variables) [44] but our method is guaranteed to recover all attractors both singletons and cyclic attractors in the case of asynchronous updates. In addition, it sheds light on Boolean organization and the role of synchronous, asynchronous, or mixed updates.

An interesting observation is the complementarity of the synchronous and mixed mode asymptotic graphs, as particularly illlustrated by the mammalian example: the mixed mode asymptotic graph recovers the general structure of the atttractors in terms of the modules' attractors, while the synchronous asymptotic graph provides details of the state sequence within an attractor. The mixed asymptotic graph can be interpreted as the graph of a network, 
Table 4: Summary of interconnection results between each pair of networks listed in Table 2. The column "Interconnections" states the input/output connections used for each pair of models. The column "Attractors" contains the total number of attractors of each module (attractors of $G^{A, u}$ and $G^{B, v}$, for all $u$, all $v$ ), and in the synchronous $\left(G^{a}\right)$ and mixed $\left(G_{m x}^{a}\right)$ strategy asymptotic graphs. The column "Size" reports the size of the synchronous and mixed asymptotic graphs, to be compared with the total number of states, $2^{N_{A} \times N_{B}}$, of the full interconnected systems.

\begin{tabular}{|c|c|c|c|c|c|c|c|c|c|}
\hline \multirow[t]{2}{*}{ Organism } & \multicolumn{2}{|c|}{ Interconnections } & \multicolumn{4}{|c|}{ Attractors } & \multicolumn{3}{|c|}{ Size } \\
\hline & $\mathrm{A} \rightarrow \mathrm{B}$ & $\mathrm{B} \rightarrow \mathrm{A}$ & $G^{A, u}$ & $G^{B, v}$ & $G^{a}$ & $G_{m x}^{a}$ & $G^{a}$ & $G_{m x}^{a}$ & $G_{-}^{I}$ \\
\hline Cyanobacteria & $\overline{C_{T} \vee C_{T S} \dashv R}$ & $\overline{C_{U} \rightarrow C_{T}}$ & 4 & 3 & 2 & 2 & 88 & 25 & $\overline{256}$ \\
\hline \multirow[t]{3}{*}{ Mammalian } & & $B M A L \dashv C y c E$ & 2 & 4 & 5 & 5 & 352 & 54 & $2^{17}$ \\
\hline & $C d c 20 \wedge C y c B$ & $B M A L \dashv C y c A$ & 2 & 4 & 8 & 5 & 472 & 54 & $2^{17}$ \\
\hline & & $B M A L \dashv C y c B$ & 2 & 4 & 7 & 5 & 424 & 54 & $2^{17}$ \\
\hline Vertebrates & $\begin{array}{c}\text { GATA3 } \rightarrow I L 10 \\
G A T A 3 \dashv S T A T 4 \\
S T A T 6 \dashv I L 18 R \\
\text { Tbet } \rightarrow I F N \gamma\end{array}$ & $I F N \gamma R \rightarrow J A K 1$ & 12 & 64 & 33 & 33 & 984 & 768 & $2^{23}$ \\
\hline Arabidopsis & $\begin{array}{c}A P C \wedge C y c B \\
\quad \dashv m R N A\end{array}$ & $\begin{array}{l}W O X 5 \dashv C y c D \\
A R R 2 \rightarrow A P C\end{array}$ & 4 & 23 & 11 & 7 & 1077 & 125 & $2^{30}$ \\
\hline
\end{tabular}

formed by an interconnection of two modules, where the dynamical timescale of each module is faster relative to the timescale of communication between modules. This property is often observed in the analysis of biological processes: fast metabolism / slow gene expression; fast post-translational processes/slow transcription.

Synchronous modules with asynchronous interconnections The dichotomy between asynchronous and synchronous networks shows that, from a dynamical point of view, the asymptotic behavior of an asynchronous network can be fully predicted by that of its component modules (any pair). In contrast, a synchronous interconnection can generate new asymptotic behavior, not predicted by its component modules (some pairs). In fact, the asynchronous strategy ensures that any partition of a network contains representatives of each basins of attraction while under the synchronous strategy some network partitions fail to represent all basins of attraction, hence some attractors are "forgotten". In other words, the asymptotic graph method is guaranteed to give exact results whenever $\mathcal{W}_{0}$ (of $G^{a}$ ) contains a representative of each basin of attraction in the synchronous case or $G_{*}^{a}$ contains a representative of each family of pathways leading to an attractor.

The dynamical behavior of large networks is less costly to evaluate with synchronous updates. Our results suggest that an interesting coupling strategy is to use the mixed asymptotic graph method: each network module is evaluated according to a synchronous updating strategy (or any other, since only the state transition graphs of the modules are needed), and then the two networks are interconnected through an asynchronous asymptotic graph, which guarantees computation of all attractors of the full network and is much smaller than a synchronous asymptotic graph (see Table 4). 
A method for analysis of emergent behaviors State-of-the-art mathematical models are presently available for a large selection of biological phenomena. One of the next steps in mathematical biology is to study the emergent dynamics arising from the coupling of two or more different models and thus predict the joints effects of two known biological processes. Our asymptotic graph method is an ideal framework to analyse emergent phenomena due to the coupling of previously studied networks. Indeed, given the state transition graphs of each module network (computed according to any updating strategy), an advantageous method is to adopt an asynchronous strategy for the coupling of the two modules. This will preserve the specific dynamics of each module and construct an asymptotic graph that recovers all the emergent behavior of the fully interconnected network. As illustrated by several examples throughout different organisms, the (mixed) asymptotic graph method is a powerful tool for studying the interaction between two known networks and predicting their joint behavior. As in the mammalian or vertebrate $\mathrm{T}$ helper cell examples, our method is also useful for interrogating models and testing new hypotheses, thereby checking the validity of the proposed interaction mechanisms.

\section{Acknowledgements}

M. Chaves was partially supported by the French agency for research through project ICycle ANR-16-CE33-0016-01. D. Figueiredo and M.A. Martins are partially supported by the ERDF European Regional Development Fund through the Operational Programme for Competitiveness and Internationalisation - COMPETE 2020 and by National Funds through the Portuguese funding agency, FCT - Fundação para a Ciência e a Tecnologia within project POCI-01-0145-FEDER-016692 and project UID/MAT/ 04106/2013 at CIDMA. D. Figueiredo acknowledges the support of FCT via the PhD scholarship PD/BD/114186/2016.

This work was partially supported by a France-Portugal partnership PHC PESSOA 2018 between M. Chaves (Campus France \#40823SD) and M.A. Martins (POCI-01-0145-FEDER030947).

\section{References}

[1] Abou-Jaoudé, W., Traynard, P., Monteiro, P., Saez-Rodriguez, J., Helikar, T., Thieffry, D., Chaouiya, C.: Logical modeling and dynamical analysis of cellular networks. Frontiers in genetics 7, 94 (2016)

[2] Akutsu, T., Melkman, A., Tamura, T., Yamamoto, M.: Determining a singleton attractor of a boolean network with nested canalyzing functions. J. Computational Biology 18(10), $1275-1290$ (2011)

[3] Baccala, R., Kono, D., Theofilopoulos, A.: Interferons as pathogenic effectors in autoimmunity. Immunological Reviews 204(9-26) (2005)

[4] Baldazzi, V., Ropers, D., Markowicz, Y., Kahn, D., Geiselmann, J., de Jong, H.: The carbon assimilation network in Escherichia coli is densely connected and largely signdetermined by directions of metabolic fluxes. PLoS Comput. Biol. 6(6), e1000,812 (2010)

[5] Chaves, M., Albert, R., Sontag, E.: Robustness and fragility of boolean models for genetic regulatory networks. J. Theoretical Biology 235(3), 431-449 (2005)

[6] Chaves, M., Carta, A.: Attractor computation using interconnected boolean networks: testing growth rate models in e. coli. Theoretical Computer Science 599, 47-63 (2015) 
[7] Chaves, M., Preto, M.: Hierarchy of models: From qualitative to quantitative analysis of circadian rhythms in cyanobacteria. Chaos 23(2), 025,113 (2013)

[8] Chaves, M., Tournier, L.: Predicting the asymptotic dynamics of large biological networks by interconnections of Boolean modules. In: Proc. $50^{\text {th }}$ Conf. Decision and Control and European Control Conf. Orlando, Florida, USA (2011)

[9] Chaves, M., Tournier, L.: Analysis tools for interconnected boolean networks with biological applications. Frontiers in Physiology 9, 586 (2018)

[10] Comet, J.P., Bernot, G., Das, A., Diener, F., Massot, C., Cessieux, A.: Simplified models for the mammalian circadian clock. Procedia Computer Science 11, 127-138 (2012)

[11] Crama, Y., Hammer, P.: Boolean functions. Theory, algorithms, and applications. Cambridge University Press, New York (2011)

[12] Demongeot, J., Elena, A., Sené, S.: Robustness in regulatory networks: A multidisciplinary approach. Acta Biotheoretica 56(1), 27-49 (2008)

[13] Demongeot, J., Goles, E., Morvan, M., Noual, M., Sené, S.: Attraction basins as gauges of robustness against boundary conditions in biological complex systems. PLOS ONE 5(8), $1-18(2010)$

[14] Devloo, V., Hansen, P., Labbé, M.: Identification of all steady states in large networks by logical analysis. Bull. Math. Biol. 65, 1025-1051 (2003)

[15] Diestel, R.: Graph Theory, $3^{\text {rd }}$ edition. Springer, Heidelberg (2005)

[16] Dong, G., Yang, Q., Wang, Q., Kim, Y.I., Wood, T., Osteryoung, K., van Oudenaarden, A., Golden, S.: Elevated atpase activity of kaic applies a circadian checkpoint on cell division in synechococcus elongatus. Cell 140, 529-539 (2010)

[17] Fauré, A., Naldi, A., Chaouiya, C., Thieffry, D.: Dynamical analysis of a generic boolean model for the control of the mammalian cell cycle. Bioinformatics 22(14), 124-131 (2006)

[18] Feillet, C., Krusche, P., Tamanini, F., Janssens, R., Downey, M., Martin, P., Teboul, M., Saito, S., Lévi, F., Bretschneider, T., van der Horst, G., Delaunay, F., Rand, D.: Phase locking and multiple oscillating attractors for the coupled mammalian clock and cell cycle. PNAS 111(27), 9828-9833 (2014)

[19] Feillet, C., van der Horst, G., Lévi, F., Rand, D., Delaunay, F.: Coupling between the circadian clock and cell cycle oscillators: Implication for healthy cells and malignant growth. Frontiers in Neurology 6, 96 (2015)

[20] García-Gomez, M., Azpeitia, E., Alvarez-Buylla, E.: A dynamic genetic-hormonal regulatory network model explains multiple cellular behaviors of the root apical meristem of Arabidopsis thaliana. PLoS Comput. Biol. 13(4), e1005,488 (2017)

[21] Garg, A., Di Cara, A., Xenarios, I., Mendoza, L., De Micheli, G.: Synchronous versus asynchronous modeling of gene regulatory networks. Bioinformatics 24(17), 1917-1925 (2008)

[22] Gerard, C., Goldbeter, A.: Temporal self-organization of the cyclin/Cdk network driving the mammalian cell cycle. PNAS 106(51), 21,643-21,648 (2009) 
[23] Hong, C., Hwang, J., Cho, K.H., Shin, I.: An efficient steady-state analysis method for large boolean networks with high maximum node connectivity. PLoS ONE 10(12), e0145,734 (2015)

[24] Irons, D.: Improving the efficiency of attractor cycle identification in Boolean networks. Physica D 217, 7-21 (2006)

[25] Jarrah, A., Raposa, B., Laubenbacher, R.: Nested canalyzing, unate cascade, and polynomial functions. Physica D 233(2), 167-174 (2007)

[26] Kauffman, S., Peterson, C., Samuelsson, B., Troein, C.: Genetic networks with canalyzing boolean rules are always stable. PNAS 101(49), 17,102-17,107 (2004)

[27] Klamt, S., Saez-Rodriguez, J., Lindquist, J., Simeoni, L., Gilles, E.: A methodology for the structural and functional analysis of signaling and regulatory networks. BMC Bioinformatics 7(56) (2006)

[28] Klarner, H., Bockmayr, A., Siebert, H.: Computing symbolic steady states of boolean networks. In: J. Was, G. Sirakoulis, S. Bandini (eds.) Cellular Automata, LNCS, vol. 8751, pp. 561-570. Springer Heidelberg (2014)

[29] Klarner, H., Siebert, H.: Approximating attractors of boolean networks by iterative ctl model checking. Frontiers in Bioengineering and Biotechnology 3 (2015)

[30] Mendoza, L., Xenarios, I.: A method for the generation of standardized qualitative dynamical systems of regulatory networks. Theoretical Biology and Medical Modelling 3(13) (2006)

[31] Mori, T., Flöttmann, M., Krantz, M., Akutsu, T., Klipp, E.: Stochastic simulation of boolean rxncon models: towards quantitative analysis of large signaling networks. BMC Systems Biology 9(45), 1-9 (2015)

[32] Ortiz-Gutiérrez, E., García-Cruz, K., Azpeitia, E., Castillo, A., Sánchez, M., AlvarezBuylla, E.: A dynamic gene regulatory network model that recovers the cyclic behavior of Arabidopsis thaliana cell cycle. PLoS Comput. Biol. 11(9), e1004,486 (2015)

[33] Plikus, M.V., Vollmers, C., de la Cruz, D., Chaix, A., Ramos, R., Panda, S., Chuong, C.M.: Local circadian clock gates cell cycle progression of transient amplifying cells during regenerative hair cycling. PNAS 110(23), E2106-E2115 (2013)

[34] Purnick, P., Weiss, R.: The second wave of synthetic biology: from modules to systems. Nature reviews. Molecular cell biology 10, 410-22 (2009)

[35] Rust, M., Markson, J., Lane, W., Fisher, D., O’Shea, E.: Ordered phosphorylation governs oscillation of a three-protein circadian clock. Science 318, 809 (2007)

[36] Saez-Rodriguez, J., Simeoni, L., Lindquist, J.A., Hemenway, R., Bommhardt, U., Arndt, B., Haus, U.U., Weismantel, R., Gilles, E.D., Klamt, S., Schraven, B.: A logical model provides insights into T cell receptor signaling. PLoS Comput. Biol. 3(8), e163 (2007)

[37] Shmulevich, I., Dougherty, E., Kim, S., Zhang, W.: Probabilistic boolean networks: a rule-based uncertainty model for gene regulatory networks. Bioinformatics 18(2), 261$274(2002)$ 
[38] Sontag, E.: Mathematical Control Theory (2nd ed.). Springer-Verlag, New York (1998)

[39] Stoll, G., Caron, B., Viara, E., Dugourd, A., Zinovyev, A., Naldi, A., Kroemer, G., Barillot, E., Calzone, L.: Maboss 2.0: an environment for stochastic boolean modeling. Bioinformatics 33(4), 2226-2228 (2017)

[40] Tournier, L., Chaves, M.: Interconnection of asynchronous Boolean networks, asymptotic and transient dynamics. Automatica 49(4), 884-893 (2013)

[41] Vecchio, D.D., Ninfa, A., Sontag, E.: Modular cell biology: Retroactivity and insulation. Molecular Systems Biology 4, 161 (2008)

[42] Veliz-Cuba, A., Aguilar, B., Hinkelmann, F., Laubenbacher, R.: Steady state analysis of boolean molecular network models via model reduction and computational algebra. BMC Bioinformatics 15, 221 (2014)

[43] Wang, R.S., Saadatpour, A., Albert, R.: Boolean modeling in systems biology: an overview of methodology and applications. Physical Biology 9, 055,001 (2012)

[44] Zañudo, J., Albert, R.: An effective network reduction approach to find the dynamical repertoire of discrete dynamic networks. Chaos 23(2), 025,111 (2013)

[45] Zhang, R., Shah, M., Yang, J., Nyland, S., Liu, X., Yun, J., Albert, R., T.P. Loughran, Jr.: Network model of survival signaling in large granular lymphocyte leukemia. PNAS 105(42), 16,308-16,313 (2008) 

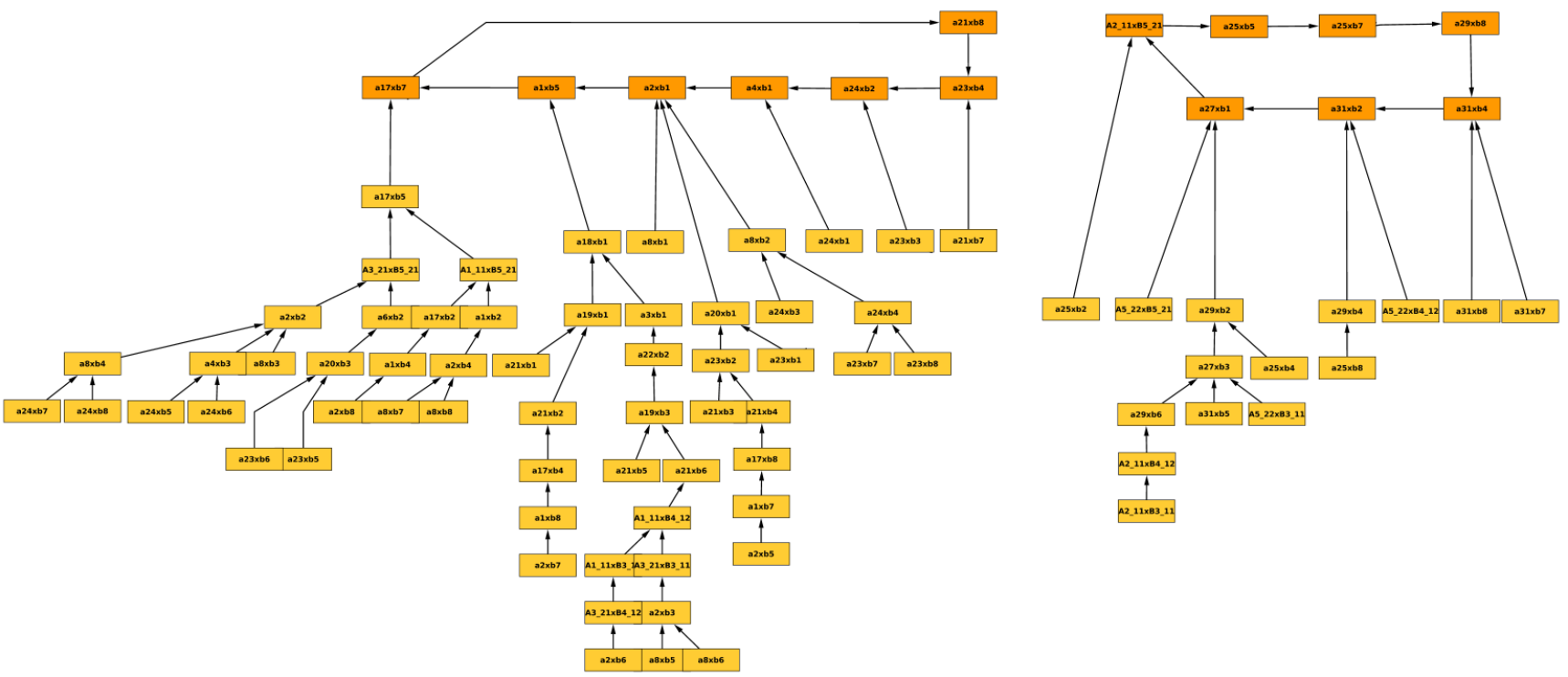

Figure 11: The synchronous asymptotic graph of the Kai proteins interconnection. Dark shaded states belong to an attractor.
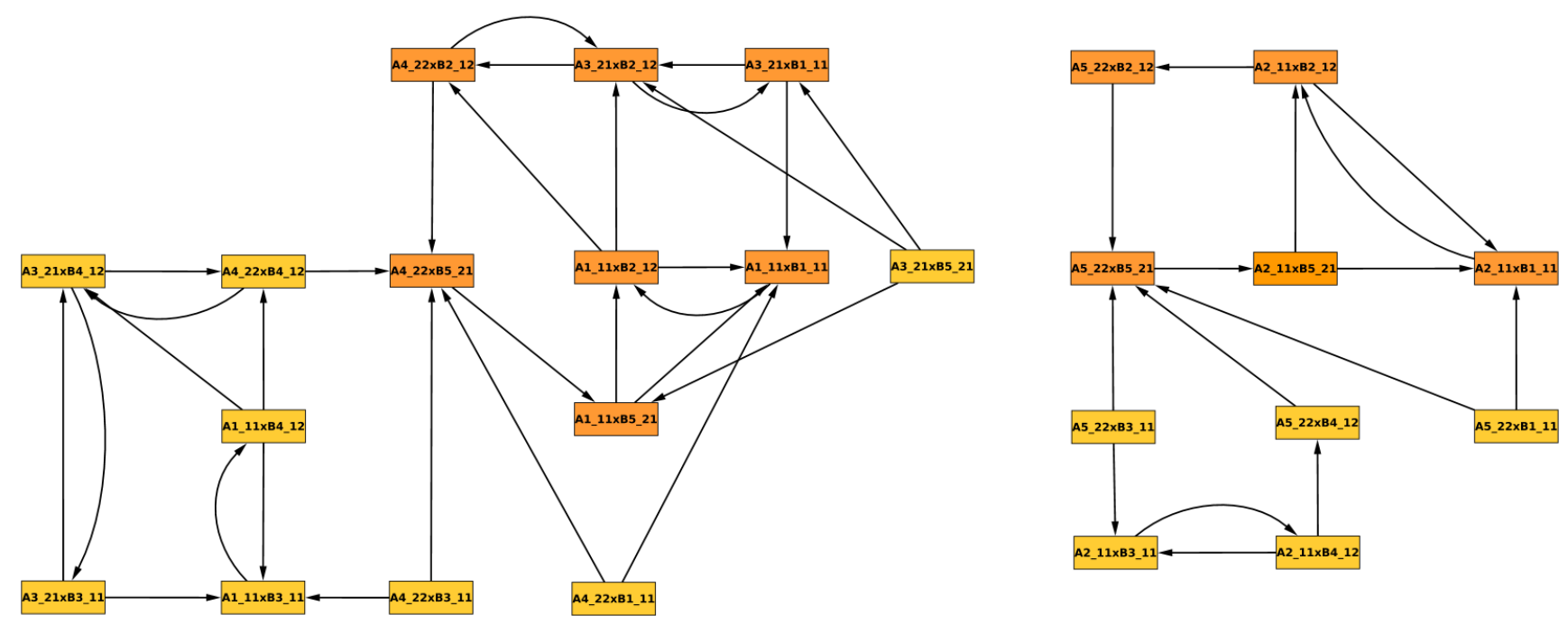

Figure 12: The mixed mode asymptotic graph of the Kai proteins interconnection. Dark shaded states belong to an attractor. 
A4_21 1 B8_12

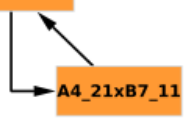

A4_21×B7_11

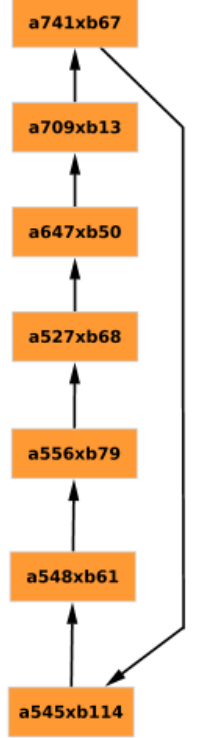

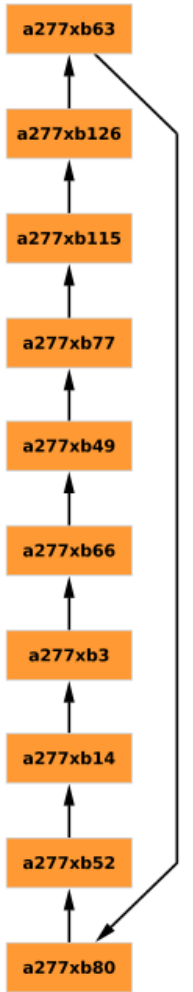

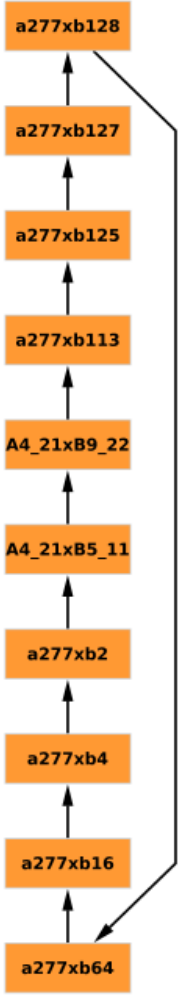

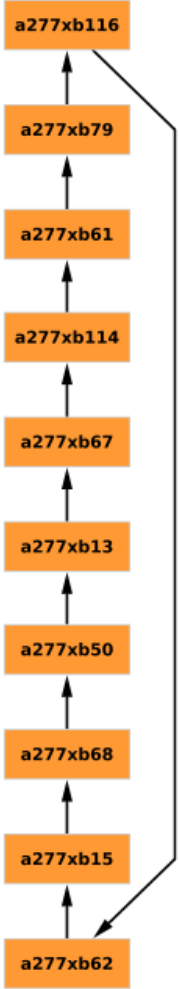

Figure 13: The five attractors of the synchronous asymptotic graph of the mammalian modules interconnection. 

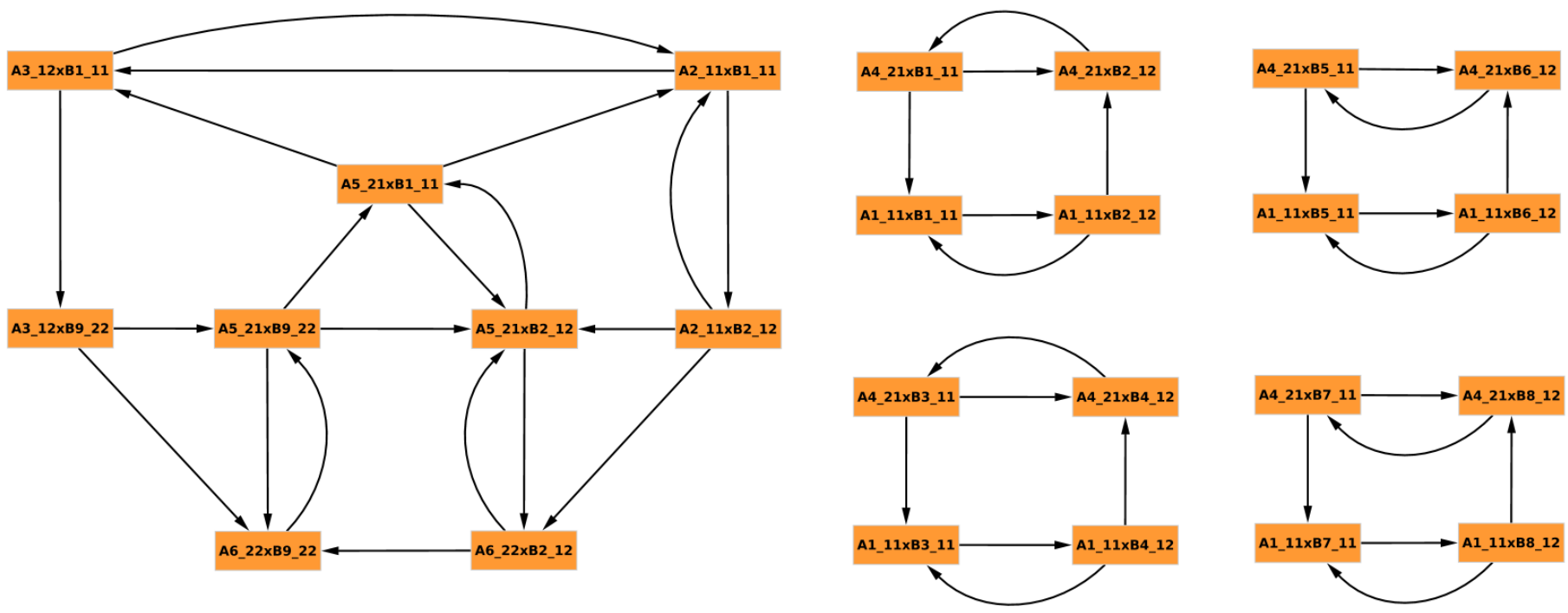

Figure 14: The five attractors of the mixed mode asymptotic graph of the mammalian modules interconnection. Left: $Q_{m x}^{2}$ 